\title{
SPECTRAL GAP \\ FOR \\ AN UNRESTRICTED KAWASAKI TYPE DYNAMICS
}

\author{
GUSTAVO POSTA
}

\begin{abstract}
We give an accurate asymptotic estimate for the gap of the generator of a particular interacting particle system. The model we consider may be informally described as follows. A certain number of charged particles moves on the segment $[1, L] \cap \mathbb{N}$ according to a Markovian law. If $\eta_{k} \in \mathbb{Z}$ is the charge at a site $k \in[1, L] \cap \mathbb{N}$ one unitary charge, positive or negative, jumps to a neighboring site, $k \pm 1$ at a rate which depends on the charge at site $k$ and at site $k \pm 1$. The total charge $\sum_{k=1}^{L} \eta_{k}$ is preserved by the dynamics, in this sense our dynamics is similar to the Kawasaki dynamics, but in our case there is no restriction on the maximum charge allowed per site. The model is equivalent to an interface dynamics connected with the stochastic Ising model at very low temperature: the "unrestricted solid on solid model". Thus the results we obtain may be read as results for this model. We give necessary and sufficient conditions to ensure that gap shrinks as $L^{-2}$, independently of the total charge. We follow the method outlined in some papers by Yau (Lu, Yau (1993), Yau (1994)) where a similar spectral gap is proved for the original Kawasaki dynamics.
\end{abstract}

\section{INTRODUCTION}

In this paper we will prove a sharp asymptotic estimate for the spectral gap of a particular interacting particle system. The system we consider may be informally described as follows. Fix $L \in \mathbb{N}$ and consider the segment $[1, L] \cap \mathbb{Z}$ in the one dimensional lattice $\mathbb{Z}$. The points of this segment will be called sites. The process we are going to study consists of a certain number of charges moving on this segment according to a Markovian law.

Suppose that to every site $k$ is attached an integer charge $\eta_{k} \in \mathbb{Z}$. A configuration of our system will be an integer valued vector $\eta \equiv\left(\eta_{1}, \ldots, \eta_{L}\right)$. For fixed $N \in \mathbb{Z}, \beta>0$ and $J \equiv\left(J_{l}, J_{r}\right) \in(0,1] \times(0,1]$, the equilibrium of the system is described by the probability measure

$$
\nu_{L}^{J, N}(\eta) \equiv \frac{1}{Z_{L}^{J, N}} \mathbf{1}(\bar{\eta}=N) e^{-\beta\left(J_{l}\left|\eta_{1}\right|+\sum_{k=2}^{L-1}\left|\eta_{k}\right|+J_{r}\left|\eta_{L}\right|\right)} .
$$

Here $Z_{L}^{J, N}$ is a normalization coefficient and $\bar{\eta} \equiv \sum_{k=1}^{L} \eta_{k}$ stands for the total charge of the configuration $\eta$. The dynamics of the system is a reversible

ESAIM: Probability and Statistics is an electronic journal with URL address http://www.emath.fr/ps/

Received by the journal March 26, 1996. Revised December 16, 1996. Accepted for publication January 3, 1997.

(c) Société de Mathématiques Appliquées et Industrielles. Typeset by $\mathrm{T}_{\mathrm{E}} \mathrm{X}$. 
continuous time Markov chain with values in $\mathbb{Z}^{L}$ and ergodic measure $\nu_{L}^{J, N}$. The chain evolves in the following way. Suppose that the system is initially in the state $\eta$, then for every site $k$, with certain rates which depends only on the charges at sites $k$ and $k \pm 1$, a unitary charge (positive or negative) jumps from the site $k$ to one of the neighboring site $k-1$ or $k+1$. This means that only transition of the type $\eta \mapsto \eta \pm \delta_{k} \mp \delta_{k+1}$ are allowed ( $\delta_{k}$ stands for the vector with all the components identically equal to zero except the $k^{\text {th }}$ which is equal to one). This dynamics obviously preserves the total charge $\bar{\eta}$ of the system, and the jump rates may be chosen so that the generator of the process is self adjoint in $L^{2}\left(\nu_{L}^{J, N}\right)$.

This kind of processes, in which the total number of particles (charges in our case) is preserved, have been studied by several authors. In particular we refer to Lu, Yau (1993), Yau (1994) and Landim, Sethuraman, Varadhan (1995) where spectral gap for similar models are computed.

In Lu, Yau (1993), for the first time, the so called martingale approach is used to prove the exact asymptotic convergence rate, i.e. $L^{-2}$, of the gap of the original Kawasaki dynamics for the Ising model in a finite cube of side $L$ in the one phase region. In this model only one particle per site is allowed. New difficulties arise if one tries to extend the proof to the case in which more than one particle per site is allowed. These difficulties are overcome in Yau (1994). Here the exact asymptotic estimate on the logarithmic Sobolev constant, and virtually on the gap, is computed for a model in which a fixed number of particles, greater than one, per site is allowed.

In Landim, Sethuraman, Varadhan (1995) using the martingale approach, a similar spectral gap is proved for a class of dynamics, the so called Zero-Range Processes. In this case a fixed number of particles moves on a discrete segment (or cube) of side $L$ and every particle jumps from a site to another site at a rate which depend only on the number of particles at the site that the particle is leaving. In this case there is not an upper bound to the number of particles allowed for any site, but because the total number of particles is fixed it is clear that the number of particles at a site can not excess this total number.

In our model the maximum charge per site is not fixed. From a technical point of view, this fact produces new difficulties in the use of the martingale approach. Moreover the asymmetry of the measure $\nu_{L}^{J, N}$ forces us to use heavily the large deviation apparatus.

The model we consider has the following physical motivation. Consider the stochastic Ising model in the cylinder $\mathcal{C} \equiv[1, L] \cap \mathbb{N} \times \mathbb{Z}$ with boundary conditions $\xi(x) \equiv \operatorname{sign}\langle\mathbf{n}, x\rangle$, where $\mathbf{n} \equiv(-N, L)$. A state $\sigma$ of this system is an element of $\Omega_{\mathcal{C}} \equiv\{-1,+1\}^{\mathcal{C}}$ and the equilibrium of the system is described by the usual Gibbs measure:

$$
\mu(\sigma) \equiv \frac{e^{-\beta H(\sigma)}}{Z(\mathcal{C})} .
$$

Here $Z(\mathcal{C})$ is a normalization factor and

$$
H(\sigma) \equiv \frac{1}{2} \sum_{\substack{|x-y|=1 \\ x, y \in \mathcal{C}}}[1-\sigma(x) \sigma(y)]+\frac{1}{2} \sum_{\substack{|x-y|=1 \\ x \in \mathcal{C}, y \in \mathcal{C}^{c}}} J(x, y)[1-\sigma(x) \xi(y)],
$$


where

$$
J(x, y) \equiv \begin{cases}J_{l} \in(0,1] & \text { if } x=\left(1, x_{2}\right), x_{2} \in \mathbb{Z} \\ J_{r} \in(0,1] & \text { if } x=\left(L, x_{2}\right), x_{2} \in \mathbb{Z}\end{cases}
$$

The function $J$ gives the interaction of the system with the border of the cylinder.

A Glauber dynamics is an $\Omega_{\mathcal{C}^{-} \text {valued Markov process with generator }}$ defined on cylindrical functions as

$$
(G f)(\sigma) \equiv \sum_{x \in \mathcal{C}} c_{x}(\sigma)\left[f\left(\sigma^{x}\right)-f(\sigma)\right]
$$

self-adjoint in $L^{2}(\mu)$. Here $\sigma^{x}$ denotes the configuration obtained from $\sigma$ by replacing the value of $\sigma$ at $x$ with its opposite.

Consider now the lattices $\mathbb{Z}^{2}$ and $\left(\mathbb{Z}^{2}\right)^{*} \equiv \mathbb{Z}^{2}+\left(\frac{1}{2}, \frac{1}{2}\right)$ as graphs embedded in $\mathbb{R}^{2}$. It is possible to associate to every configuration $\sigma \in \Omega_{\mathcal{C}}$ a polygonal in $\mathbb{R}^{2}$ in the following way. Call bond every unitary segment connecting two points of $\left(\mathbb{Z}^{2}\right)^{*}$ and site every point in $\mathbb{Z}^{2}$. Then we say that two sites $x$ and $y$ in $\mathbb{Z}^{2}$ are separated by the bond $h$ if their Euclidean distance from $h$ is equal to $\frac{1}{2}$. Given $\sigma \in \Omega_{\mathcal{C}}$ we denote by $P(\sigma) \subset \mathbb{R}^{2}$ the collection of all bonds separating sites $x$ and $y$ in $\mathbb{Z}^{2}$ where $\sigma(x) \neq \sigma(y)$. If moreover we use the convention that any pair of orthogonal bonds that intersects in a given site $x^{*}$ of the dual lattice $\left(\mathbb{Z}^{2}\right)^{*}$ are a linked pair of bonds if they are both on the same side of the forty-five degrees line across $x^{*}$, then we immediately see that $P(\sigma)$ splits up in a unique way in a collection of finite closed contours $\gamma_{1}(\sigma), \ldots, \gamma_{n}(\sigma)$, and a unique infinite open contour or interface $\Gamma(\sigma)$. The correspondence between configurations and contours we obtain in this way is $1-1$. An open contour $\Gamma$ is said to be admissible if there exists $\sigma \in \Omega_{\mathcal{C}}$ such that $\Gamma=\Gamma(\sigma)$. It is possible to write explicitly the probability to have a fixed admissible contour $\Gamma$ using the low temperature cluster expansion (see Dobrushin, Kontecký, Shlosman (1992)), if we assume for simplicity that $J_{l}=J_{r}=1$ (being the general case easy to figure out) we have:

$$
\mu(\sigma: \Gamma(s)=\Gamma)=\frac{\exp [-2 \beta|\Gamma|+W(\beta, \Gamma)]}{Z(\mathcal{C})} .
$$

Here $|\Gamma|$ is the length, i.e. the number of bonds, of $\Gamma$ and $W(\beta, \Gamma)$ is a cluster term. This cluster term becomes small for large values of $\beta$.

The study of the evolution of $\Gamma$ under the Markovian dynamics (1.1) is not an easy task. In order to attack the problem one can simplify the model by supposing that:

i) $\Gamma$ is the graph of an integer valued function $\Phi$;

ii) there are no closed contours.

These are natural assumption in the limit $\beta \rightarrow+\infty$. The model we obtain is the so called one dimensional solid on solid model. The equilibrium measure of the model is defined on the set of all function $\Phi:[1, L] \cap \mathbb{N} \rightarrow \mathbb{Z}$ as:

$$
\mu_{L}^{J, N}(\Phi)=\frac{1}{Z_{L}^{J, N}(\beta)} e^{-\beta\left(J_{l}|\Phi(1)|+\sum_{k=1}^{L-1}|\Phi(k+1)-\Phi(k)|+J_{r}|N-\Phi(L)|\right)},
$$


while the dynamics of the system is a reversible continuous time Markov chain with values in $\mathbb{Z}^{L}$ and ergodic measure $\mu_{L}^{J, N}$. The chain evolves in the following way. Suppose that the system is initially in the state $\Phi$, then for every $k=1, \ldots, L$, with certain rates which depends only on $\Phi(k-1)$ and $\Phi(k+1)$, the system evolves in one of the two configurations $\Phi \pm \delta_{k}$. The transition rates are chosen so that the generator of the process is self adjoint in $L^{2}\left(\mu_{L}^{J, N}\right)$.

Notice that for any $\Phi \equiv\left(\Phi_{1}, \ldots, \Phi_{L}\right) \in \mathbb{Z}^{L}$ we have

$$
\begin{aligned}
& \nu_{L+1}^{J, N}\left(\eta_{1}=\Phi_{1}, \eta_{1}+\eta_{2}=\Phi_{2} \ldots, \eta_{1}+\cdots+\eta_{L}=\Phi_{L}\right)= \\
= & \nu_{L+1}^{J, N}\left(\eta_{1}=\Phi_{1}, \eta_{2}=\Phi_{2}-\Phi_{1}, \ldots, \eta_{L}=\Phi_{L}-\Phi_{L-1}\right)=\mu_{L}^{J, N}(\Phi) .
\end{aligned}
$$

Moreover if we define for $\eta \in \mathbb{Z}^{L+1}$ the random variable $\Phi(\eta) \equiv\left(\eta_{1}, \eta_{1}+\right.$ $\left.\eta_{2}, \ldots, \eta_{1}+\cdots+\eta_{L}\right) \in \mathbb{Z}^{L}$ it simple to check that $\Phi\left(\eta \pm \delta_{k} \mp \delta_{k+1}\right)=\Phi(\eta) \pm \delta_{k}$. Thus our particles system with jumping charges is equivalent to the solid on solid model, and the results we will obtain may be read as results about this model.

The solid on solid model is a good approximation of the Ising model for large values of $\beta$. However in this paper we do not investigate the connection between the solid on solid model and the Ising model.

\section{Notation and Results}

Our sample space is $\Omega_{L} \equiv \mathbb{Z}^{L}$ for fixed $L \in \mathbb{N}$. Sometimes it will be useful to consider the infinite product space $\Omega \equiv \mathbb{Z}^{\mathbb{N}}$. Configurations, i.e. , elements of the sample space $\left(\Omega_{L}\right.$ or $\Omega$ ) will be denoted by greek letters, e.g. $\eta=$ $\left(\eta_{1}, \ldots, \eta_{L}\right) \in \Omega_{L}$. If $f$ is a real function on $\Omega_{L}$, are defined the following discrete derivatives:

$$
\begin{aligned}
\left(\partial_{i}^{+} f\right)(\eta) & =f\left(\eta+\delta_{i}\right)-f(\eta) \\
\left(\partial_{i}^{-} f\right)(\eta) & =f\left(\eta-\delta_{i}\right)-f(\eta) \\
\left(\partial_{i, j} f\right)(\eta) & =f\left(\eta+\delta_{i}-\delta_{j}\right)-f(\eta),
\end{aligned} \quad i, j=1, \ldots, L
$$

A function $f: \Omega \rightarrow \mathbb{R}$ is local in $k \in \mathbb{N}$ if $f \equiv f\left(\eta_{k}\right)$. For every $U \subset \mathbb{N}$ and $\eta \in \Omega_{L}$ or $\eta \in \Omega, \eta_{U}$ stands for the restriction of $\eta$ to $U$. The minimal $\sigma$-field for which are measurable the functions:

$$
\left\{\eta \in \Omega \mapsto \eta_{U} \in \mathbb{Z}^{|U|}: U \subset \mathbb{N},|U|<+\infty\right\}
$$

is denoted by $\mathcal{F}$. The restriction of $\mathcal{F}$ to $\Omega_{L}$ will be denoted by $\mathcal{F}_{L}$. Finally if $\left\{g_{i}: i \in I\right\}$ is a family of function indexed on a set of finite cardinality $|I|$, the symbol $\operatorname{Av}_{i \in I} g_{i}$ stands for the arithmetic mean $\frac{1}{|I|} \sum_{i \in I} g_{i}$ of the family.

Given $N \in \mathbb{Z}, L \in \mathbb{N}, \beta>0$ and $J \equiv\left(J_{l}, J_{r}\right) \in(0,1] \times(0,1]$ one defines the probability measure $\nu_{L}^{J, N}$ on $\left(\Omega_{L}, \mathcal{F}_{L}\right)$ as:

$$
\nu_{L}^{J, N}(\eta) \equiv \frac{1}{Z_{L}^{J, N}} \mathbf{1}(\bar{\eta}=N) e^{-\beta\left(J_{l}\left|\eta_{1}\right|+\sum_{k=2}^{L-1}\left|\eta_{k}\right|+J_{r}\left|\eta_{L}\right|\right)}
$$


where

$$
Z_{L}^{J, N} \equiv \sum_{\eta \in \Omega_{L}} \mathbf{1}(\bar{\eta}=N) e^{-\beta\left(J_{l}\left|\eta_{1}\right|+\sum_{k=2}^{L-1}\left|\eta_{k}\right|+J_{r}\left|\eta_{L}\right|\right)}
$$

It is elementary to check that this definition is correct, i.e. that $Z_{L}^{J, N}<+\infty$. Expectation with respect to $\nu_{L}^{J, N}$ is set as $\mathbf{E}_{L}^{J, N}(\cdot)$, while variance is set as $\operatorname{Var}_{L}^{J, N}(\cdot)$. Now we define our process. This is a Markov process with infinitesimal generator $G_{L}^{J, N}$ defined by its action on cylinder functions $f$ as:

$$
\left(G_{L}^{J, N} f\right)(\eta) \equiv \sum_{\xi} c_{L}^{J, N}(\eta, \xi)[f(\xi)-f(\eta)]
$$

where:

$$
c_{L}^{J, N}(\eta, \xi) \equiv \begin{cases}\left(\frac{\nu_{I}^{J, N}(\xi)}{\nu_{L}^{J, N}(\eta)}\right)^{\frac{1}{2}} & \begin{array}{l}
\text { if } \nu_{L}^{J, N}(\eta)>0 \text { and } \xi=\eta \pm \delta_{k} \mp \delta_{k+1} \\
\text { for some } k=1, \ldots, L-1 \\
0 \text { elsewhere. }
\end{array}\end{cases}
$$

It is a simple to verify that these rates are uniformly bounded in $N$ and $L$ and that $G_{L}^{J, N}$ is self-adjoint in $L^{2}\left(\nu_{L}^{J, N}\right)$, it is negative definite and its largest eigenvalue is 0 . The process defined by the generator $G_{L}^{J, N}$ is a reversible, irreducible ergodic Markov chain with ergodic measure $\nu_{L}^{J, N}$. The spectral gap of the process is defined as the absolute value of the largest negative eigenvalue of the generator: $\lambda_{1}\left(G_{L}^{J, N}\right) \equiv-\sup \left\{\lambda \in \operatorname{Spec}\left(G_{L}^{J, N}\right): \lambda<0\right\}$. The gap can be also characterized as:

$$
\lambda_{1}\left(G_{L}^{J, N}\right)=\inf _{f \in L^{2}\left(\nu_{L}^{J, N}\right)} \frac{\mathcal{G}_{L}^{J, N}(f, f)}{\operatorname{Var}_{L}^{J, N}(f)}
$$

where $\mathcal{G}_{L}^{J, N}$ is the Dirichlet form associated with the generator $G_{L}^{J, N}$.

We are now in a position to state the main results of this paper:

Theorem 2.1. Suppose that $\delta \in(0,1)$ and define $J=(\delta, 1)$. Then there exists $K_{1}(\beta, \delta)$ and $K_{2}(\beta, \delta)>0$ such that:

$$
K_{1} L^{-2} \leq \lambda_{1}\left(G_{L}^{J, N}\right) \leq K_{2} L^{-2}
$$

for every $L>0$ and $N \in \mathbb{Z}$.

Two corollaries follow from this theorem:

Corollary 2.2. Suppose that $0<J_{l}<J_{r}<1$ and define $J=\left(J_{l}, J_{r}\right)$. Then there exists $K_{1}\left(\beta, J_{l}, J_{r}\right)$ and $K_{2}\left(\beta, J_{l}, J_{r}\right)$ such that:

$$
K_{1} L^{-2} \leq \lambda_{1}\left(G_{L}^{J, N}\right) \leq K_{2} L^{-2}
$$

for every $L>0$ and $N \in \mathbb{Z}$.

Corollary 2.3. Suppose that $\delta \in(0,1)$ and define $J=(\delta, \delta)$. Then for every $N \in \mathbb{Z}$ there exists $K_{1}(\beta, \delta, N)$ and $K_{2}(\beta, \delta, N)$ such that:

$$
K_{1} L^{-2} \leq \lambda_{1}\left(G_{L}^{J, N}\right) \leq K_{2} L^{-2} .
$$


for every $L>0$.

REMARK 2.1. The approach we present here to prove a spectral gap estimate is adapted from Lu and Yau's method in Lu, Yau (1993) and Yau (1994). The method works properly when the spectral gap is independent of the number of particles $N$ as in Corollary 2.2, but it does not when the gap depend on the number of particles as in Corollary 2.3. Since the constants in (2.4) are dependent on $N$ and we will not study this dependence, this is a very poor result. However this result still has a physical motivation; for $N=0$ it gives the correct bound for the gap of the solid on solid model (see Section 1) with horizontal interface.

It is interesting to understand physically why the symmetric case is so different from the asymmetric one.

A way to understand this problem is to think to the configurations $\eta$ which are "typical" for the measure $\nu_{L}^{J, N}$. In order to determinate these configurations, we have to minimize the energy $J_{l}\left|\eta_{1}\right|+\sum_{k=2}^{L-1}\left|\eta_{k}\right|+J_{r}\left|\eta_{L}\right|$ under the constrain $\eta_{1}+\ldots+\eta_{L}=N$. It is easy to check that the solution to this problem in the case $J_{l}=J_{r}$ is given by the configurations $\eta$ such that $\eta_{L}=N-\eta_{1}, \eta_{1}=0, \ldots, N$ and $\eta_{k}=0$ for $k=2, \ldots, L-1$. This says us that the energy landscape has, in this case, a "plateau" in its minimum. The cardinality of the platea $u$ is proportional to $N$, this suggest that the spectral gap of the process should depend on $N$. This fact does not happen in the asymmetric case. In fact if for example $J_{l}<J_{r}$, then the minimum of the energy $J_{l}\left|\eta_{1}\right|+\sum_{k=2}^{L-1}\left|\eta_{k}\right|+J_{r}\left|\eta_{L}\right|$ under the constrain $\eta_{1}+\ldots+\eta_{L}=N$ is attained only by the configuration $\eta=(N, 0, \ldots, 0)$. So in this case there is no plateau and the gap does not depend on $N$.

The above discussion gives information about the solid on solid interface $\Phi$. In the symmetric case the "typical interface" is flat and stays over the $x$ axis at an height between 0 and $N$. In the asymmetric case the typical interface is again flat, but it prefer to stay at height $N$ over the $x$ axis.

REMARK 2.2. The asymmetry introduced by $\delta<1$ in Theorem 2.1 is essential in proving a one site spectral gap of $\nu_{L}^{\delta, N}$ which does not depend on the total charge $N$, as we will see in Section 6 . This uniform estimate on the one site spectral gap is one of the basic technical tool to use the Lu and Yau method (Lu, Yau (1993) and Yau (1994)), as it will be clear by reading Section 3 . In the symmetric case $\delta=1$ the one site spectral gap does depend on $N$.

The same problem, i.e. how to prove a one site spectral gap, is also in Landim, Sethuraman, Varadhan (1995), but because in that case the model is a finite state Markov chain, the solution is simpler.

\section{Preliminary Results}

The proof of the results stated in Section 2 unfortunately requires heavy technical preliminaries. The aim of this section is to give a concise list of this results in the hope that this will make more readable the next section in which the main results are proved. The results stated in the present section will be proved in sections 6,7 and 8 . 
We are particularly interested in the study of $\nu_{L}^{J, N}$ for $J=(\delta, 1)$ and $\delta \in(0,1]$. In order to simplify the notation we will write $\nu_{L}^{\delta, N}$ instead of $\nu_{L}^{J, N}$. In the particular case of $\delta=1$ we will omit the index $1: \nu_{L}^{N} \equiv \nu_{L}^{\delta, N}$. The same index notation is used for mean variance etc.

The first result we present is a one site Poincaré inequality. This result and others related to the one site marginal of $\nu_{L}^{J, N}$ will be proved in Section 6 . Proposition 3.1 (One Site Spectral Gap). Suppose that $\delta \in(0,1)$, $\bar{\rho}>0$ and $M \in(0,+\infty]$. Then:

i) There exists $K_{1}(\beta, \bar{\delta}, \bar{\rho})$ and $\bar{L}(\beta, \delta, \bar{\rho})>0$ such that:

$$
\operatorname{Var}_{L}^{\delta, N}\left(f|| \eta_{1} \mid \leq M\right) \leq K_{1} L \mathbf{E}_{L}^{\delta, N}\left[\left(\partial_{1}^{+} f\right)^{2}|| \eta_{1} \mid \leq M\right]
$$

for every $L>\bar{L}, N \in \mathbb{Z}$ with $|N / L| \leq \bar{\rho}$ and for every $f \in L^{2}\left(\nu_{L}^{\delta, N}\right)$ local in 1.

ii) There exists $K_{2}(\beta, \delta)>0$, such that:

$$
\operatorname{Var}_{L}^{\delta, N}(f) \leq K_{2} \mathbf{E}_{L}^{\delta, N}\left[\left(\partial_{k}^{+} f\right)^{2}\right]
$$

for every $L>0, N \in \mathbb{Z}, k=2, \ldots, L$ and for every $f \in L^{2}\left(\nu_{L}^{\delta, N}\right)$ local in $k$. The next two lemmas treat the large deviation of the one site marginal of $\nu_{L}^{\delta, N}$

Lemma 3.2. Suppose that $\delta \in(0,1)$. Then there exists $\bar{\rho}(\beta, \delta), K_{1}(\beta, \delta)$ and $K_{2}(\beta, \delta)>0$ such that:

$$
\nu_{L}^{\delta, N}\left(\eta_{1}<0\right) \leq K_{1} \sqrt{L} e^{-K_{2} L},
$$

for every $L>0$ and $N>\bar{\rho} L$, while:

$$
\nu_{L}^{\delta, N}\left(\eta_{1}>0\right) \leq K_{1} \sqrt{L} e^{-K_{2} L}
$$

for every $N<-\bar{\rho} L$.

Lemma 3.3. Suppose that $\delta \in(0,1]$ and fix $\bar{\rho}>0$. Then for every $M>\bar{\rho}$ there exists $K_{1}(\beta, \delta)$ and $K_{2}(\beta, \delta, \bar{\rho}, M)>0$ such that:

$$
\nu_{L}^{\delta, N}\left(\left|\eta_{1}\right|>M L\right) \leq K_{1} \sqrt{L} e^{-K_{2} L}
$$

for every $L>0$ and $N \in \mathbb{Z}$ with $|N / L| \leq \bar{\rho}$.

The following proposition is of very technical nature. It is close to a similar result obtained by Yau in Yau (1994), in the simpler context of bounded random variables and it is one of the key ingredient in the proof of Theorem 2.1. Define the quadratic form

$$
\mathcal{E}_{L}^{\delta, N}(f, f) \equiv \sum_{k=1}^{L-1} \mathbf{E}_{L}^{\delta, N}\left[\left(\partial_{k+1, k} f\right)^{2}\right]
$$

then: 
Proposition 3.4 (Two Block Estimate). Suppose that $\delta=1, \bar{\rho}>0$ and define for a bounded real function $h$ the random variable $h_{j} \equiv h\left(\eta_{j}\right)$ for $j=1, \ldots, L$. Then for every $\varepsilon>0$ there exists $K(\beta, \varepsilon)$ and $\bar{L}(\beta, \varepsilon, \bar{\rho})>0$ such that:

$$
\mathbf{E}_{L}^{N}\left(f, \mathrm{Av}_{j} h_{j}\right)^{2} \leq K \mathcal{E}_{L}^{N}(f, f)+\frac{\varepsilon}{L} \operatorname{Var}_{L}^{N}(f)
$$

for every $f \in L^{2}\left(\nu_{L}^{N}\right), L>\bar{L}$ and $N \in \mathbb{Z}$ with $|N / L| \leq \bar{\rho}$.

Here and later $\mathbf{E}_{L}^{\delta, N}(f, g)$ denotes the covariance of $f$ and $h$ with respect to $\nu_{L}^{\delta, N}$.

The following two proposition will be used in the next sections as a starting point for an inductive procedure. The first one assure us that if $J=(\delta, 1)$ with $\delta \in(0,1)$ the generator $G_{L}^{J, N}$ exhibits a positive spectral gap uniformly in $N$. The second proposition tell us only that if $J=(1,1)$ the spectral gap of the generator $G_{L}^{J, N}$ is positive.

Proposition 3.5. Suppose $\delta \in(0,1)$. Then there exists $K(\beta, \delta, L)>0$ such that:

$$
\operatorname{Var}_{L}^{\delta, N}(f) \leq K \mathcal{E}_{L}^{\delta, N}(f, f)
$$

for every $N \in \mathbb{Z}, L>0$ and $f \in L^{2}\left(\nu_{L}^{\delta, N}\right)$.

Proposition 3.6. There exists $K(\beta, N, L)>0$ such that:

$$
\operatorname{Var}_{L}^{N}(f) \leq K \mathcal{E}_{L}^{N}(f, f),
$$

for every $L>0, N \in \mathbb{Z}$ and $f \in L^{2}\left(\nu_{L}^{N}\right)$.

\section{Proof of Main Results: Lower Bound}

In this section we will prove the first and more difficult inequality in (2.2). The proof we present is adapted from the proof of a similar result proved in Yau (1994).

It is important to understand the following obvious property of $\nu_{L}^{\delta, N}$ :

$$
\begin{aligned}
& \nu_{L}^{\delta, N}\left(\xi \mid \eta_{i}, \eta_{i+1}, \ldots, \eta_{L}\right)= \\
= & \mathbf{1}\left(\xi_{i}=\eta_{i}, \ldots, \xi_{L}=\eta_{L}\right) \nu_{i-1}^{\delta, N-\eta_{i}-\cdots-\eta_{L}}\left(\xi_{1}, \ldots, \xi_{i-1}\right)
\end{aligned}
$$

for every $i>1$. This property will be used without any comment in the sequel, especially in the form:

$$
\mathbf{E}_{L}^{\delta, N}\left(f \mid \eta_{i}, \eta_{i+1}, \ldots, \eta_{L}\right)=\mathbf{E}_{i-1}^{\delta, N-\eta_{i}-\cdots-\eta_{L}}\left[f\left(\cdot \mid \eta_{i}, \eta_{i+1}, \ldots, \eta_{L}\right)\right] .
$$

The notation $f\left(\cdot \mid \eta_{i}, \eta_{i+1}, \ldots, \eta_{L}\right)$ stresses the fact that $\eta_{i}, \ldots, \eta_{L}$ should be considered as parameters: the expectation in (4.1) is taken only on the variables $\eta_{1}, \ldots, \eta_{i-1}$. Remember that

$$
\mathcal{E}_{L}^{\delta, N}(f, f) \equiv \sum_{k=1}^{L-1} \mathbf{E}_{L}^{\delta, N}\left[\left(\partial_{k+1, k} f\right)^{2}\right],
$$


using the characterization (2.1), reversibility and the bounds on the rates it is simple to prove that:

$$
e^{-\beta} \inf _{f \in L^{2}\left(\nu_{L}^{\delta, N}\right)} \frac{\mathcal{E}_{L}^{\delta, N}(f, f)}{\operatorname{Var}_{L}^{\delta, N}(f)} \leq \lambda_{1}\left(G_{L}^{J, N}\right) \leq e^{\beta} \inf _{f \in L^{2}\left(\nu_{L}^{\delta, N}\right)} \frac{\mathcal{E}_{L}^{\delta, N}(f, f)}{\operatorname{Var}_{L}^{\delta, N}(f)}
$$

where $\delta \in(0,1)$ and $J=(\delta, 1)$. We are going to use the first of these inequalities to prove our lower bound on the gap. In order to make simpler the notations in this section we suppose that $\beta>0$ and $\delta \in(0,1)$ are fixed constant. When we speak about constants in this section these constants may depend on $\beta$ and $\delta$. However if the constants depend on $L$ or $N$ this fact is explicitly mentioned.

Define:

$$
V(L) \equiv \sup _{\substack{f \in L^{2}\left(\nu_{L}^{\delta, N}\right) \\ N \in \mathbb{Z}}} \frac{\operatorname{Var}_{L}^{\delta, N}(f)}{\mathcal{E}_{L}^{\delta, N}(f, f)}
$$

for $L>0$. By Proposition $3.5 V(L)<+\infty$. The aim of this section is to show that

$$
\sup _{L>0} \frac{V(L)}{L^{2}}<+\infty \text {. }
$$

This yields immediately $(2.2)$.

The idea is to prove (4.2) recursively. This is done in the following fundamental proposition:

Proposition 4.1. There exists a positive constant $K$ such that:

$$
\begin{aligned}
V(2 L) & \leq 2 V(L)+K L^{2} \\
V(2 L+1) & \leq 2 V(L)+K L^{2},
\end{aligned}
$$

for every $L>0$.

From this lemma the bound (4.2) follows easily. In fact, for $n \in \mathbb{N}$, define

$$
W_{n} \equiv \sup _{L \in\left[2^{n}, 2^{n+1}\right)} \frac{V(L)}{L^{2}},
$$

and notice that (4.2) is equivalent to prove that the sequence $\left\{W_{n}: n \in \mathbb{N}\right\}$ is bounded above by a constant. Let $L_{n} \in\left[2^{n}, 2^{n+1}\right) \cap \mathbb{N}$ be such that $W_{n}=\frac{V\left(L_{n}\right)}{L_{n}^{2}}$. Using Proposition 4.1 it is easy to check that:

$$
W_{n+1}=\frac{V\left(L_{n+1}\right)}{L_{n+1}^{2}} \leq \frac{1}{2} W_{n}+\frac{K}{4} \leq V(1)+K \leq K^{\prime} .
$$

Proof of Proposition 4.1. We will prove (4.3), the proof of the other estimate (4.4) being similar.

The general strategy of the proof is to show that for every $\varepsilon \in(0,1)$ there exists $C(\varepsilon)$ and $\bar{L}(\varepsilon)>0$ such that for every $L>\bar{L}$ we have:

$$
\operatorname{Var}_{2 L}^{\delta, N}(f) \leq V(L) \mathcal{E}_{2 L}^{\delta, N}(f, f)+C(\varepsilon) L^{2} \mathcal{E}_{2 L}^{\delta, N}(f, f)+\varepsilon \operatorname{Var}_{2 L}^{\delta, N}(f)
$$


taking $\varepsilon=1 / 2$ in the previous estimate we obtain:

$$
\frac{\operatorname{Var}_{2 L}^{\delta, N}(f)}{\mathcal{E}_{2 L}^{\delta, N}(f, f)} \leq 2 V(L)+2 C L^{2}
$$

i.e. (4.3). The proof of (4.5) is divided into several steps for purposes of clarity.

Fix $L \in \mathbb{N}$ and define the subsets $\left\{\alpha_{j}: j=L, \ldots, 2 L+1\right\}$ of $\mathbb{N}$ as:

$$
\alpha_{j}= \begin{cases}\{1, \ldots, 2 L\} & \text { if } j=L \\ \{j, \ldots, 2 L\} & \text { if } L<j<2 L+1 \\ \emptyset & \text { if } j=2 L+1\end{cases}
$$

STEP 1. Suppose that $f \in L^{2}\left(\nu_{2 L}^{\delta, N}\right)$ and define $f_{j} \equiv \mathbf{E}_{2 L}^{\delta, N}\left(f \mid \eta_{\alpha_{j}}\right)$. Then:

$$
\operatorname{Var}_{2 L}^{\delta, N}(f) \leq V(L) \mathcal{E}_{2 L}^{\delta, N}(f, f)+\sum_{j=L+1}^{2 L} \mathbf{E}_{2 L}^{\delta, N}\left[\operatorname{Var}_{2 L}^{\delta, N}\left(f_{j} \mid \eta_{\alpha_{j+1}}\right)\right]
$$

Proof of Step 1. A simple computation shows that:

$$
\begin{aligned}
& \operatorname{Var}_{2 L}^{\delta, N}(f)= \\
= & \mathbf{E}_{2 L}^{\delta, N}\left[\operatorname{Var}_{2 L}^{\delta, N}\left(f \mid \eta_{\alpha_{L+1}}\right)\right]+\sum_{j=L+1}^{2 L} \mathbf{E}_{2 L}^{\delta, N}\left[\operatorname{Var}_{2 L}^{\delta, N}\left(f_{j} \mid \eta_{\alpha_{j+1}}\right)\right] .
\end{aligned}
$$

Because $\operatorname{Var}_{2 L}^{\delta, N}\left(f \mid \eta_{\alpha_{L+1}}\right)=\operatorname{Var}_{L}^{\delta, N-\bar{\eta}_{\alpha_{L}+1}}\left[f\left(\cdot \mid \eta_{\alpha_{L}+1}\right)\right]$ this term is bounded above by

$$
\begin{aligned}
& V(L) \mathcal{E}_{L}^{\delta, N-\bar{\eta}_{\alpha_{L+1}}}\left[f\left(\cdot \mid \eta_{\alpha_{L}+1}\right), f\left(\cdot \mid \eta_{\alpha_{L}+1}\right)\right]= \\
= & V(L) \sum_{k=1}^{L-1} \mathbf{E}_{2 L}^{\delta, N}\left[\left(\partial_{k+1, k} f\right)^{2} \mid \eta_{\alpha_{L}+1}\right] .
\end{aligned}
$$

This implies:

$$
\mathbf{E}_{2 L}^{\delta, N}\left[\operatorname{Var}_{2 L}^{\delta, N}\left(f \mid \eta_{\alpha_{L+1}}\right)\right] \leq V(L) \mathcal{E}_{2 L}^{\delta, N}(f, f)
$$

Now substituting this estimate into (4.7) we obtain (4.6).

To obtain (4.5) we shall bound each term in the sum on the right hand side of (4.6) with terms proportional to $\mathcal{E}_{2 L}^{\delta, N}(f, f)$ and terms proportional to the variance of $f$. More precisely we would like to prove an inequality of the form:

$$
\mathbf{E}_{2 L}^{\delta, N}\left[\operatorname{Var}_{2 L}^{\delta, N}\left(f_{j} \mid \eta_{\alpha_{j+1}}\right)\right] \leq C(\varepsilon) L \mathcal{E}_{2 L}^{\delta, N}(f, f)+\frac{\varepsilon}{L} \operatorname{Var}_{2 L}^{\delta, N}(f)
$$

The next step is in this direction: 
STEP 2. Suppose that $L+1 \leq j \leq 2 L$. Then there exists $K$ and $\bar{\rho}>0$ such that if $B_{j}$ is the set defined by $B_{j} \equiv\left\{\eta \in \Omega_{2 L}:\left|N-\bar{\eta}_{\alpha_{j}}\right| \leq \bar{\rho} L\right\}$ the following inequality holds:

$$
\begin{aligned}
& \mathbf{E}_{2 L}^{\delta, N}\left[\operatorname{Var}_{2 L}^{\delta, N}\left(f_{j} \mid \eta_{\alpha_{j+1}}\right)\right] \leq K L \mathcal{E}_{2 L}^{\delta, N}(f, f)+\varepsilon(L) \operatorname{Var}_{2 L}^{\delta, N}(f)+ \\
+ & K \mathbf{E}_{2 L}^{\delta, N}\left[\mathbf{1}\left(B_{j}\right) \mathbf{E}_{2 L}^{\delta, N}\left(f, \operatorname{Av}_{i=2}^{j-1} g_{i} \mid \eta_{\alpha_{j}}\right)^{2}\right] .
\end{aligned}
$$

Here $\varepsilon(L)=o\left(L^{-1}\right)$ and $g_{i}(\eta) \equiv \Gamma_{i}\left(N, \eta_{\alpha_{j}}\right) e^{-\beta\left(\left|\eta_{i}-1\right|-\left|\eta_{i}\right|\right)}$, where $\Gamma_{i}\left(N, \eta_{\alpha_{j}}\right)$ is a bounded positive function.

Proof of Step 2. Recall that $\operatorname{Var}_{2 L}^{\delta, N}\left(f_{j} \mid \eta_{\alpha_{j+1}}\right)=\operatorname{Var}_{j}^{\delta, N-\bar{\eta}_{\alpha_{j+1}}}\left[f_{j}\left(\cdot \mid \eta_{\alpha_{j+1}}\right)\right]$. Because $f_{j}$ is a function of only $\eta_{\alpha_{j}}$, then for $\eta_{\alpha_{j+1}}$ fixed, $f_{j}\left(\cdot \mid \eta_{\alpha_{j+1}}\right)$ is a function of only $\eta_{j}$, i.e. is local in $j>L$. We can use the "one site spectral gap" (3.2) to bound $\operatorname{Var}_{2 L}^{\delta, N}\left(f_{j} \mid \eta_{\alpha_{j+1}}\right)$ :

$$
\operatorname{Var}_{2 L}^{\delta, N}\left(f_{j} \mid \eta_{\alpha_{j+1}}\right) \leq C_{1} \mathbf{E}_{2 L}^{\delta, N}\left[\left(\partial_{j}^{+} f_{j}\right)^{2} \mid \eta_{\alpha_{j+1}}\right]
$$

Now we need to transform the Glauber type gradient on right hand side of (4.10) in a Kawasaki type gradient. An elementary calculation shows that:

$$
\left(\partial_{j}^{+} f_{j}\right)(\eta)=-\mathbf{E}_{2 L}^{\delta, N}\left(\partial_{j, i} f \mid \eta_{\alpha_{j}}+\delta_{j}\right)+\mathbf{E}_{2 L}^{\delta, N}\left(f, g_{i} \mid \eta_{\alpha_{j}}\right)
$$

for every $i=1, \ldots, j-1$. Here $g_{i}$ is defined as:

$$
g_{i}(\eta)=\frac{\nu_{2 L}^{\delta, N}\left(\eta-\delta_{i}+\delta_{j} \mid \eta_{\alpha_{j+1}}\right)}{\nu_{2 L}^{\delta, N}\left(\eta \mid \eta_{\alpha_{j+1}}\right)} \frac{\nu_{2 L}^{\delta, N}\left(\eta_{j} \mid \eta_{\alpha_{j+1}}\right)}{\nu_{2 L}^{\delta, N}\left(\eta_{j} \mid \eta_{\alpha_{j+1}}+\delta_{j}\right)}
$$

It is simple to check that:

$$
\begin{aligned}
& g_{1}(\eta)=\Gamma_{1}\left(N, \eta_{\alpha_{j}}\right) e^{-\beta \delta\left(\left|\eta_{1}-1\right|-\left|\eta_{1}\right|\right)} \\
& g_{i}(\eta)=\Gamma_{i}\left(N, \eta_{\alpha_{j}}\right) e^{-\beta\left(\left|\eta_{i}-1\right|-\left|\eta_{i}\right|\right)} \quad i=2, \ldots, j-1,
\end{aligned}
$$

where $\Gamma_{i}$ are bounded positive functions. Notice that the left hand side of (4.11) does not depend on $i$. Averaging over $i=2, \ldots, j-1$ this expression yields:

$$
\left(\partial_{j}^{+} f_{j}\right)(\eta)=-\mathbf{E}_{2 L}^{\delta, N}\left(\operatorname{Av}_{i=2}^{j-1}\left(\partial_{j, i} f\right) \mid \eta_{\alpha_{j}}+\delta_{j}\right)+\mathbf{E}_{2 L}^{\delta, N}\left(f, \mathrm{Av}_{i=2}^{j-1} g_{i} \mid \eta_{\alpha_{j}}\right)
$$

Define $B_{j}=\left\{\eta \in \Omega_{2 L}:\left|N-\bar{\eta}_{\alpha_{j}}\right| \leq \bar{\rho} L\right\}$, where $\bar{\rho}$ is a positive constant to be fixed later. From (4.11), (4.13) and some simple estimates we obtain:

$$
\begin{aligned}
& \mathbf{E}_{2 L}^{\delta, N}\left[\left(\partial_{j}^{+} f_{j}\right)^{2}\right] \leq C_{2}\left\{\mathbf{E}_{2 L}^{\delta, N}\left[\left(\partial_{j, 1} f\right)^{2}+\operatorname{Av}_{i=2}^{j-1}\left(\partial_{j, i} f\right)^{2}\right]+\right. \\
+ & \mathbf{E}_{2 L}^{\delta, N}\left[\mathbf{1}\left(B_{j}^{c}\right) \mathbf{E}_{2 L}^{\delta, N}\left(f, g_{1} \mid \eta_{\alpha_{j}}\right)^{2}\right]+ \\
+ & \left.\mathbf{E}_{2 L}^{\delta, N}\left[\mathbf{1}\left(B_{j}\right) \mathbf{E}_{2 L}^{\delta, N}\left(f, \mathrm{Av}_{i=2}^{j-1} g_{i} \mid \eta_{\alpha_{j}}\right)^{2}\right]\right\}
\end{aligned}
$$


The three terms on the right hand side of this expression correspond to the three term on the right hand side of (4.9). The last one is exactly the same, while the first two can be easily transformed. We start estimating the first one.

It is elementary to check that $\left(\partial_{j, i} f\right)=\sum_{k=i}^{j-1}\left(\partial_{k+1, k} f\right)\left(\eta^{j, k+1}\right)$, where $\eta^{s, t} \equiv \eta-\delta_{s}+\delta_{t}$. This implies:

$$
\begin{aligned}
& \mathbf{E}_{2 L}^{\delta, N}\left[\left(\partial_{j, i} f\right)^{2}\right] \leq 2 L \sum_{k=1}^{L-1} \mathbf{E}_{2 L}^{\delta, N}\left[\left(\partial_{k+1, k} f\right)^{2}\left(\eta^{j, k+1}\right)\right] \leq \\
\leq & C_{3} L \mathcal{E}_{2 L}^{\delta, N}(f, f),
\end{aligned}
$$

which gives the first term on the right hand side of (4.9).

For the second term on the right hand side of (4.14) we can use the Schwarz inequality and (4.12) to prove that there exists a positive constant $C_{4}$ such that:

$$
\begin{aligned}
& \mathbf{1}\left(B_{j}^{c}\right) \mathbf{E}_{2 L}^{\delta, N}\left(f, g_{1} \mid \eta_{\alpha_{j}}\right)^{2} \leq \\
\leq & C_{4} \mathbf{1}\left(B_{j}^{c}\right) \nu_{2 L}^{\delta, N}\left(\eta_{1}<1 \mid \eta_{\alpha_{j}}\right) \nu_{2 L}^{\delta, N}\left(\eta_{1} \geq 1 \mid \eta_{\alpha_{j}}\right) .
\end{aligned}
$$

By Lemma 3.2 we know there exists $\bar{\rho}>0$ such that

$$
\nu_{2 L}^{\delta, N}\left(\eta_{1}<1 \mid \eta_{\alpha_{j}}\right) \nu_{2 L}^{\delta, N}\left(\eta_{1} \geq 1 \mid \eta_{\alpha_{j}}\right)=o\left(L^{-1}\right)
$$

if $\left|N-\eta_{\alpha_{j}}\right|>\bar{\rho} L$. This fact and the estimate (4.16) imply that:

$$
\mathbf{1}\left(B_{j}^{c}\right) \mathbf{E}_{2 L}^{\delta, N}\left(f, g_{1} \mid \eta_{\alpha_{j}}\right)^{2} \leq \varepsilon(L) \operatorname{Var}_{2 L}^{\delta, N}\left(f \mid \eta_{\alpha_{j}}\right)
$$

where $\varepsilon(L)=o\left(L^{-1}\right)$.

The next step to obtain (4.8) is to bound the last term of (4.9). The basic idea is to use on this term the "two block estimate" (3.4). Notice that to apply this result we need identically distributed random variables with bounded density $\rho \equiv \frac{1}{L} \sum_{i=1}^{L} \eta_{i}$. The naive way to obtain identically distributed variables is to condition the covariance term on the left hand side in (4.9) with respect to $\eta_{1}$. Before doing so, in order to have a bounded density, we have to bound above $\left|\eta_{1}\right|$.

STEP 3. There exists $K$ and $M(\bar{\rho})>0$ such that:

$$
\begin{aligned}
& \mathbf{E}_{2 L}^{\delta, N}\left[\mathbf{1}\left(B_{j}\right) \mathbf{E}_{2 L}^{\delta, N}\left(f, \mathrm{Av}_{i=2}^{j-1} g_{i} \mid \eta_{\alpha_{j}}\right)^{2}\right] \leq \varepsilon(L) \operatorname{Var}_{2 L}^{\delta, N}(f)+ \\
+ & K \mathbf{E}_{2 L}^{\delta, N}\left[\mathbf{1}\left(B_{j}\right) \mathbf{E}_{2 L}^{\delta, N}\left(f, \mathbf{1}(A) \mathrm{Av}_{i=2}^{j-1} g_{i} \mid \eta_{\alpha_{j}}\right)^{2}\right]
\end{aligned}
$$

where $\varepsilon(L)=o\left(L^{-1}\right)$ and $A \equiv\left\{\eta \in \Omega_{2 L}:\left|\eta_{1}\right| \leq M L\right\}$.

Proof of Step 3. A simple calculation shows that:

$$
\begin{aligned}
& \mathbf{E}_{2 L}^{\delta, N}\left(f, \mathrm{Av}_{i=2}^{j-1} g_{i} \mid \eta_{\alpha_{j}}\right)^{2} \leq \\
\leq & 2 \mathbf{E}_{2 L}^{\delta, N}\left(f, \mathbf{1}(A) \mathrm{Av}_{i=2}^{j-1} g_{i} \mid \eta_{\alpha_{j}}\right)^{2}+2\|g\|_{+\infty}^{2} \operatorname{Var}_{2 L}^{\delta, N}\left(f \mid \eta_{\alpha_{j}}\right) \nu_{2 L}^{\delta, N}\left(A^{c} \mid \eta_{\alpha_{j}}\right) .
\end{aligned}
$$


But by Lemma 3.3, we know that there exists $M$ so that

$$
\mathbf{1}\left(B_{j}\right) \nu_{2 L}^{\delta, N}\left(A^{c} \mid \eta_{\alpha_{j}}\right)=o\left(L^{-1}\right)
$$

This and a trivial estimate concludes the proof of (4.17).

In the next step we will condition with respect to $\eta_{1}$ the last term on the right hand side of (4.17) and we will use the "two block estimate". This will produce some "good terms" (the first three term on the right hand side of (4.18) and an "extra term" (the last term on the right hand side of (4.18)) which will be estimated later.

STEP 4. For every $\varepsilon>0$ there exists $K(\varepsilon, \bar{\rho})>0$ such that:

$$
\begin{aligned}
& \mathbf{E}_{2 L}^{\delta, N}\left[\mathbf{1}\left(B_{j}\right) \mathbf{E}_{2 L}^{\delta, N}\left(f, \mathbf{1}(A) \mathrm{Av}_{i=2}^{j-1} g_{i} \mid \eta_{\alpha_{j}}\right)^{2}\right] \leq \\
\leq & K L \mathcal{E}_{2 L}^{\delta, N}(f, f)+\frac{\varepsilon}{L} \operatorname{Var}_{2 L}^{\delta, N}(f)+\varepsilon(L) \operatorname{Var}_{2 L}^{\delta, N}(f)+ \\
+ & 16 \mathbf{E}_{2 L}^{\delta, N}\left\{\mathbf{1}\left(B_{j}\right) \operatorname{Var}_{2 L}^{\delta, N}\left[\mathbf{E}_{2 L}^{\delta, N}\left(f \mid \eta_{1}, \eta_{\alpha_{j}}\right) \mid A, \eta_{\alpha_{j}}\right] \times\right. \\
\times & \left.\operatorname{Var}_{2 L}^{\delta, N}\left[\mathbf{E}_{2 L}^{\delta, N}\left(g_{2} \mid \eta_{1}, \eta_{\alpha_{j}}\right) \mid A, \eta_{\alpha_{j}}\right]\right\},
\end{aligned}
$$

where $\varepsilon(L)=o\left(L^{-1}\right)$.

Proof of Step 4. It is elementary to check that:

$$
\begin{aligned}
& \mathbf{E}_{2 L}^{\delta, N}\left(f, \mathbf{1}(A) \mathrm{Av}_{i=2}^{j-1} g_{i} \mid \eta_{\alpha_{j}}\right)= \\
= & \mathbf{E}_{2 L}^{\delta, N}\left[\mathbf{E}_{2 L}^{\delta, N}\left(f, \mathbf{1}(A) \mathrm{Av}_{i=2}^{j-1} g_{i} \mid \eta_{1}, \eta_{\alpha_{j}}\right) \mid \eta_{\alpha_{j}}\right]+ \\
+ & \mathbf{E}_{2 L}^{\delta, N}\left[\mathbf{E}_{2 L}^{\delta, N}\left(f \mid \eta_{1}, \eta_{\alpha_{j}}\right), \mathbf{1}(A) \mathrm{Av}_{i=2}^{j-1} \mathbf{E}_{2 L}^{\delta, N}\left(g_{i} \mid \eta_{1}, \eta_{\alpha_{j}}\right) \mid \eta_{\alpha_{j}}\right] .
\end{aligned}
$$

Because $g_{i}$ is a function of $\eta_{i}$ and $\eta_{\alpha_{j}}$ only,

$$
\mathbf{E}_{2 L}^{\delta, N}\left(g_{i} \mid \eta_{1}, \eta_{\alpha_{j}}\right)=\mathbf{E}_{2 L}^{\delta, N}\left(g_{2} \mid \eta_{1}, \eta_{\alpha_{j}}\right)
$$

for every $i=2, \ldots, j-1$. Thus the term on the left hand side of (4.18) is bounded above by

$$
\begin{aligned}
& 2 \mathbf{E}_{2 L}^{\delta, N}\left[\mathbf{1}\left(A \cap B_{j}\right) \mathbf{E}_{2 L}^{\delta, N}\left(f, \mathrm{Av}_{i=2}^{j-1} g_{i} \mid \eta_{1}, \eta_{\alpha_{j}}\right)^{2}\right]+ \\
+ & 2 \mathbf{E}_{2 L}^{\delta, N}\left\{\mathbf{1}\left(B_{j}\right) \mathbf{E}_{2 L}^{\delta, N}\left[\mathbf{E}_{2 L}^{\delta, N}\left(f \mid \eta_{1}, \eta_{\alpha_{j}}\right), \mathbf{1}(A) \mathbf{E}_{2 L}^{\delta, N}\left(g_{2} \mid \eta_{1}, \eta_{\alpha_{j}}\right) \mid \eta_{\alpha_{j}}\right]^{2}\right\} .
\end{aligned}
$$

By Proposition 3.4 we know that for every $\varepsilon>0$ if $L$ is large enough, $\left|N-\bar{\eta}_{\alpha_{j}}\right| \leq \bar{\rho} L$ and $\left|\eta_{1}\right| \leq M L$ :

$$
\begin{aligned}
& \mathbf{E}_{2 L}^{\delta, N}\left(f, \mathrm{Av}_{i=2}^{j-1} g_{i} \mid \eta_{1}, \eta_{\alpha_{j}}\right)^{2}=\mathbf{E}_{j-2}^{N-\eta_{1}-\bar{\eta}_{\alpha_{j}}}\left[f\left(\cdot \mid \eta_{1}, \eta_{\alpha_{j}}\right), \mathrm{Av}_{i=2}^{j-1} g_{i}\right]^{2} \leq \\
\leq & C_{1}(\varepsilon) \mathcal{E}_{j-2}^{N-\eta_{1}-\bar{\eta}_{\alpha_{j}}}\left[f\left(\cdot \mid \eta_{1}, \eta_{\alpha_{j}}\right), f\left(\cdot \mid \eta_{1}, \eta_{\alpha_{j}}\right)\right]+ \\
+ & \frac{2 \varepsilon}{L} \operatorname{Var}_{j-2}^{N-\eta_{1}-\bar{\eta}_{\alpha_{j}}}\left[f\left(\cdot \mid \eta_{1}, \eta_{\alpha_{j}}\right)\right] .
\end{aligned}
$$


In conclusion the first term on the right hand side of (4.19) is bounded above by $2 C_{1} \mathcal{E}_{2 L}^{\delta, N}(f, f)+\frac{4 \varepsilon}{L} \operatorname{Var}_{2 L}^{\delta, N}(f)$ for every $\varepsilon>0$ and $L$ large enough. To bound the second term on the right hand side of (4.19) an elementary estimate (see Lemma 9.1) shows that:

$$
\begin{aligned}
& \mathbf{E}_{2 L}^{\delta, N}\left[\mathbf{E}_{2 L}^{\delta, N}\left(f \mid \eta_{1}, \eta_{\alpha_{j}}\right), \mathbf{1}(A) \mathbf{E}_{2 L}^{\delta, N}\left(g_{2} \mid \eta_{1}, \eta_{\alpha_{j}}\right) \mid \eta_{\alpha_{j}}\right]^{2} \leq \\
\leq & 8\left\{\mathbf{E}_{2 L}^{\delta, N}\left[\mathbf{E}_{2 L}^{\delta, N}\left(f \mid \eta_{1}, \eta_{\alpha_{j}}\right), \mathbf{E}_{2 L}^{\delta, N}\left(g_{2} \mid \eta_{1}, \eta_{\alpha_{j}}\right) \mid A, \eta_{\alpha_{j}}\right]^{2}+\right. \\
+ & \left.\|g\|_{+\infty}^{2} \nu_{2 L}^{\delta, N}\left(A^{c} \mid \eta_{\alpha_{j}}\right) \operatorname{Var}_{2 L}^{\delta, N}\left[\mathbf{E}_{2 L}^{\delta, N}\left(f \mid \eta_{1}, \eta_{\alpha_{j}}\right) \mid \eta_{\alpha_{j}}\right]\right\} .
\end{aligned}
$$

By Lemma 3.3 we know that $\nu_{2 L}^{\delta, N}\left(A^{c} \mid \eta_{\alpha_{j}}\right)=o\left(L^{-1}\right)$ for $\left|N-\bar{\eta}_{\alpha_{j}}\right| \leq \bar{\rho} L$. Thus by the Schwarz inequality the second term on the right hand side of (4.19) is bounded above by:

$$
\begin{aligned}
& 16 \mathbf{E}_{2 L}^{\delta, N}\left\{\mathbf{1}\left(B_{j}\right) \operatorname{Var}_{2 L}^{\delta, N}\left[\mathbf{E}_{2 L}^{\delta, N}\left(f \mid \eta_{1}, \eta_{\alpha_{j}}\right) \mid A, \eta_{\alpha_{j}}\right] \times\right. \\
\times & \left.\operatorname{Var}_{2 L}^{\delta, N}\left[\mathbf{E}_{2 L}^{\delta, N}\left(g_{2} \mid \eta_{1}, \eta_{\alpha_{j}}\right) \mid A, \eta_{\alpha_{j}}\right]\right\}+\varepsilon(L) \operatorname{Var}_{2 L}^{\delta, N}(f)
\end{aligned}
$$

where $\varepsilon(L)=o\left(L^{-1}\right)$. This and the previous estimates concludes the proof of (4.18).

The first three terms on right hand side in (4.18) don't need further investigation. Last term contains a variance product. Because $\eta_{\alpha_{i}}$ is fixed in the conditional expectation, $\mathbf{E}_{2 L}^{\delta, N}\left(f \mid \eta_{1}, \eta_{\alpha_{j}}\right)$ and $\mathbf{E}_{2 L}^{\delta, N}\left(g_{2} \mid \eta_{1}, \eta_{\alpha_{j}}\right)$ are local functions in $\eta_{1}$. We will use the Poincaré inequality (3.1) to bound this term. STEP 5. There exists $K(\bar{\rho})>0$ such that:

$$
\begin{aligned}
& \mathbf{E}_{2 L}^{\delta, N}\left\{\mathbf{1}\left(B_{j}\right) \operatorname{Var}_{2 L}^{\delta, N}\left[\mathbf{E}_{2 L}^{\delta, N}\left(f \mid \eta_{1}, \eta_{\alpha_{j}}\right) \mid A, \eta_{\alpha_{j}}\right] \times\right. \\
\times & \left.\operatorname{Var}_{2 L}^{\delta, N}\left[\mathbf{E}_{2 L}^{\delta, N}\left(g_{2} \mid \eta_{1}, \eta_{\alpha_{j}}\right) \mid A, \eta_{\alpha_{j}}\right]\right\} \leq \\
\leq & K \mathbf{E}_{2 L}^{\delta, N}\left[\mathbf{1}\left(A \cap B_{j}\right)\left(\partial_{1}^{+} \mathbf{E}_{2 L}^{\delta, N}\left(f \mid \eta_{1}, \eta_{\alpha_{j}}\right)\right)^{2}\right] .
\end{aligned}
$$

Proof of Step 5. By the one site spectral gap (3.1) there exists a positive constant $C_{1}(\bar{\rho})$ such that:

$$
\begin{aligned}
& \operatorname{Var}_{2 L}^{\delta, N}\left[\mathbf{E}_{2 L}^{\delta, N}\left(f \mid \eta_{1}, \eta_{\alpha_{j}}\right) \mid A, \eta_{\alpha_{j}}\right] \leq \\
\leq & C_{1} L \mathbf{E}_{2 L}^{\delta, N}\left[\left(\partial_{1}^{+} \mathbf{E}_{2 L}^{\delta, N}\left(f \mid \eta_{1}, \eta_{\alpha_{j}}\right)\right)^{2} \mid A, \eta_{\alpha_{j}}\right]
\end{aligned}
$$

and

$$
\begin{aligned}
& \operatorname{Var}_{2 L}^{\delta, N}\left[\mathbf{E}_{2 L}^{\delta, N}\left(g_{2} \mid \eta_{1}, \eta_{\alpha_{j}}\right) \mid A, \eta_{\alpha_{j}}\right] \leq \\
\leq & C_{1} L \mathbf{E}_{2 L}^{\delta, N}\left[\left(\partial_{1}^{+} \mathbf{E}_{2 L}^{\delta, N}\left(g_{2} \mid \eta_{1}, \eta_{\alpha_{j}}\right)\right)^{2} \mid A, \eta_{\alpha_{j}}\right]
\end{aligned}
$$


if $\left|N-\bar{\eta}_{\alpha_{j}}\right| \leq \bar{\rho} L$. Notice that $\mathbf{E}_{2 L}^{\delta, N}\left(g_{2} \mid \eta_{1}, \eta_{\alpha_{j}}\right)$ depends only on the density $\frac{N-\eta_{1}-\bar{\eta}_{\alpha_{j}}}{j-2}$ because $g_{2}$ is local in $\eta_{2}$. From this it should be clear that:

$$
\partial_{1}^{+} \mathbf{E}_{2 L}^{\delta, N}\left(g_{2} \mid \eta_{1}, \eta_{\alpha_{j}}\right)=O\left(L^{-1}\right)
$$

this fact is formally proved in Lemma 9.2. In conclusion:

$$
\begin{aligned}
& \mathbf{1}\left(B_{j}\right) \operatorname{Var}_{2 L}^{\delta, N}\left[\mathbf{E}_{2 L}^{\delta, N}\left(f \mid \eta_{1}, \eta_{\alpha_{j}}\right) \mid A, \eta_{\alpha_{j}}\right] \operatorname{Var}_{2 L}^{\delta, N}\left[\mathbf{E}_{2 L}^{\delta, N}\left(g_{2} \mid \eta_{1}, \eta_{\alpha_{j}}\right) \mid A, \eta_{\alpha_{j}}\right] \leq \\
\leq & C_{3}(\bar{\rho}) \mathbf{E}_{2 L}^{\delta, N}\left[\left(\partial_{1}^{+} \mathbf{E}_{2 L}^{\delta, N}\left(f \mid \eta_{1}, \eta_{\alpha_{j}}\right)\right)^{2} \mid A, \eta_{\alpha_{j}}\right] .
\end{aligned}
$$

Since $\nu_{2 L}^{\delta, N}\left(A \mid \eta_{\alpha_{j}}\right) \geq 1 / 2$ for large $L$ and $\left|N-\eta_{\alpha_{j}}\right| \leq \bar{\rho} L$ (see Lemma 3.3), (4.20) is proved.

It remains to estimate the gradient term on the right hand side of (4.20). We will use the same technique we used in Step 2.

STEP 6 . For every $\varepsilon>0$ there exist a positive constant $K(\varepsilon, \bar{\rho})$ such that:

$$
\begin{aligned}
& \mathbf{E}_{2 L}^{\delta, N}\left[\mathbf{1}\left(A \cap B_{j}\right)\left(\partial_{1}^{+} \mathbf{E}_{2 L}^{\delta, N}\left(f \mid \eta_{1}, \eta_{\alpha_{j}}\right)\right)^{2}\right] \leq \\
\leq & K L \mathcal{E}_{2 L}^{\delta, N}(f, f)+\frac{\varepsilon}{L} \operatorname{Var}_{2 L}^{\delta, N}(f) .
\end{aligned}
$$

Proof of Step 6. An elementary calculation shows that:

$$
\partial_{1}^{+} \mathbf{E}_{2 L}^{\delta, N}\left(f \mid \eta_{1}, \eta_{\alpha_{j}}\right)=-\mathbf{E}_{2 L}^{\delta, N}\left(\partial_{1, i} f \mid \eta_{1}+1, \eta_{\alpha_{j}}\right)+\mathbf{E}_{2 L}^{\delta, N}\left(f, g_{i} \mid \eta_{1}, \eta_{\alpha_{j}}\right)
$$

for every $i=2, \ldots, j-1$. This expression and some trivial estimates yield:

$$
\begin{aligned}
& \mathbf{E}_{2 L}^{\delta, N}\left[\mathbf{1}\left(A \cap B_{j}\right)\left(\partial_{1}^{+} \mathbf{E}_{2 L}^{\delta, N}\left(f \mid \eta_{1}, \eta_{\alpha_{j}}\right)\right)^{2}\right] \leq C_{1}\left\{\mathbf{E}_{2 L}^{\delta, N}\left[\operatorname{Av}_{i=2}^{j-1}\left(\partial_{1, i} f\right)^{2}\right]+\right. \\
+ & \left.\mathbf{E}_{2 L}^{\delta, N}\left[\mathbf{1}\left(A \cap B_{j}\right) \mathbf{E}_{2 L}^{\delta, N}\left(f, \mathrm{Av}_{i=2}^{j-1} g_{i} \mid \eta_{1}, \eta_{\alpha_{j}}\right)^{2}\right]\right\}
\end{aligned}
$$

for a positive constant $C_{1}$. Now, the same argument used in Step 2 to prove (4.15) can be used here to estimate the first term on the right hand side of (4.22). We obtain:

$$
\mathbf{E}_{2 L}^{\delta, N}\left[\operatorname{Av}_{i=2}^{j-1}\left(\partial_{1, i} f\right)^{2}\right] \leq C_{2} L \mathcal{E}_{2 L}^{\delta, N}(f, f)
$$

It remains to estimate the second term on the right hand side of (4.22). Fix $\varepsilon>0$, if $L>0$ is large enough the two block estimate (3.4) says that:

$$
\mathbf{E}_{2 L}^{\delta, N}\left(f, \operatorname{Av}_{i=2}^{j-1} g_{i} \mid \eta_{1}, \eta_{\alpha_{j}}\right)^{2} \leq C_{3}(\varepsilon) \mathcal{E}_{2 L}^{\delta, N}\left(f, f \mid \eta_{1}, \eta_{\alpha_{j}}\right)+\frac{\varepsilon}{L} \operatorname{Var}_{2 L}^{\delta, N}\left(f \mid \eta_{1}, \eta_{\alpha_{j}}\right)
$$

for $\left|\eta_{1}\right| \leq M L$ and $\left|N-\bar{\eta}_{\alpha_{j}}\right| \leq \bar{\rho} L$. From (4.22), (4.23) and (4.24) we obtain (4.21). 
We are finally in a position to prove (4.5). By (4.9), (4.17), (4.18), (4.20) and (4.21) for every $\varepsilon>0$ there exists $C(\varepsilon)$ and $\bar{L}(\varepsilon)>0$ such that for any $L>\bar{L}$ the following inequality holds:

$$
\mathbf{E}_{2 L}^{\delta, N}\left[\operatorname{Var}_{2 L}^{\delta, N}\left(f_{j} \mid \eta_{\alpha_{j+1}}\right)\right] \leq C(\varepsilon) L \mathcal{E}_{2 L}^{\delta, N}(f, f)+\frac{\varepsilon}{L} \operatorname{Var}_{2 L}^{\delta, N}(f)
$$

From this estimate and (4.6) we have:

$$
\operatorname{Var}_{2 L}^{\delta, N}(f) \leq V(L) \mathcal{E}_{2 L}^{\delta, N}(f, f)+C(\varepsilon) L^{2} \mathcal{E}_{2 L}^{\delta, N}(f, f)+\varepsilon \operatorname{Var}_{2 L}^{\delta, N}(f)
$$

i.e. (4.5), which implies (4.3) for $L \geq \bar{L}$. By adjusting the constant $K$ and recalling that by Proposition 3.5

$$
\sup _{L \leq \bar{L}} \sup _{N, f} \frac{\operatorname{Var}_{L}^{\delta, N}(f)}{\mathcal{E}_{L}^{\delta, N}(f, f)}=K^{\prime}(\bar{L})<+\infty,
$$

(4.3) is proved for every $L>0$.

The same proof we used to prove (4.3) may be used to prove (4.4). We only need to replace the index $2 L$ with $2 L+1$. We will omit this tedious repetition. This concludes the proof of Proposition 4.1.

\section{Proof of Main Results: Upper Bound \& Generalizations}

In this section we complete the proof of Theorem 2.1 and we prove corollaries 2.2 and 2.3. The proofs of the corollaries are similar to the proof of Theorem 2.1, so we will skip most of technicalities.

The next result gives the correct upper bound on the spectral gap of $G_{L}^{J, N}$ and concludes the proof of Theorem 2.1 .

Proposition 5.1. Suppose that $\delta \in(0,1)$. Then there exists $K_{1}(\beta, \delta)>0$ such that:

$$
\inf _{f \in L^{2}\left(\nu_{L}^{\delta, N}\right)} \frac{\mathcal{E}_{L}^{\delta, N}(f, f)}{\operatorname{Var}_{L}^{\delta, N}(f)} \leq \frac{K_{1}}{L^{2}}
$$

for every $L>0$ and $N \in \mathbb{Z}$.

Suppose that $\delta=1$. Then there exists $K_{2}(\beta)>0$ such that:

$$
\inf _{f \in L^{2}\left(\nu_{L}^{N}\right)} \frac{\mathcal{E}_{L}^{N}(f, f)}{\operatorname{Var}_{L}^{N}(f)} \leq \frac{K_{2}}{L^{2} \vee N^{2}}
$$

for every $L>0$ and $N \in \mathbb{Z}$.

Proof. We will first prove (5.2). We will show that for every $L>0$ and $N \in \mathbb{Z}$ there exists a function $F_{L} \in L^{2}\left(\nu_{L}^{N}\right)$ such that:

$$
\frac{\mathcal{E}_{L}^{N}\left(F_{L}, F_{L}\right)}{\operatorname{Var}_{L}^{N}\left(F_{L}\right)} \leq \frac{C}{L^{2} \vee N^{2}},
$$


where $C(\beta)$ is a positive constant. Direct calculation shows that for every $L \in \mathbb{N}$ there exists $g(L)$ such that:

$$
\sum_{\substack{i, j=1 \\ i \neq j}}^{L}\left(\frac{i}{L}-g(L)\right)\left(\frac{j}{L}-g(L)\right)=0 .
$$

Furthermore $g(L) \rightarrow 1 / 2$ if $L \rightarrow+\infty$. If we define

$$
F_{L}(\eta) \equiv \sum_{k=1}^{L}\left(\frac{k}{L}-g(L)\right) \eta_{k}
$$

a straightforward calculation shows that $\left(\partial_{k+1, k} F_{L}\right)(\eta)=-\frac{1}{L}$. This implies that $\mathcal{E}_{L}^{N}\left(F_{L}, F_{L}\right)=\frac{1}{L}$. From property (5.3) and some simple estimates it follows that:

$$
\operatorname{Var}_{L}^{N}\left(F_{L}\right)=\operatorname{Var}_{L}^{N}\left(\eta_{1}\right) \sum_{k=1}^{L}\left(\frac{k}{L}-g(L)\right)^{2} \geq C_{1} L \operatorname{Var}_{L}^{N}\left(\eta_{1}\right)
$$

where $C_{1}$ is a positive constant. To complete the proof of (5.2) it remains to prove that $\operatorname{Var}_{L}^{N}\left(\eta_{1}\right) \geq C_{2}\left(\frac{N^{2}}{L^{2}} \vee 1\right)$. This simple estimate is proved in the appendix (Lemma 9.3).

We now turn to the case $\delta \in(0,1)$. We will use the same test function $F_{L}$. Obviously $\mathcal{E}_{L}^{\delta, N}\left(F_{L}, F_{L}\right)=\frac{1}{L}$, so we need only to estimate the variance of $F_{L}$. A simple estimate yields:

$$
\operatorname{Var}_{L}^{\delta, N}\left(F_{L}\right) \geq \mathbf{E}_{L}^{\delta, N}\left[\operatorname{Var}_{L}^{\delta, N}\left(F_{L} \mid \eta_{1}\right)\right]
$$

Because $\operatorname{Var}_{L}^{\delta, N}\left(F_{L} \mid \eta_{1}\right)=\operatorname{Var}_{L-1}^{N-\eta_{1}}\left(F_{L-1}\right),(5.1)$ follows from (5.2).

We conclude this section proving corollaries 2.2 and 2.3 .

Proof of Corollary 2.2. For $J=\left(J_{l}, J_{r},\right)$ define the quadratic form

$$
\mathcal{E}_{L}^{J, N}(f, f)=\sum_{k=1}^{L-1} \mathbf{E}_{L}^{J, N}\left[\left(\partial_{k+1, k} f\right)^{2}\right] .
$$

As we did in the proof of Theorem 2.1 we claim that to prove Corollary 2.2 it suffices to show that

$$
\frac{K_{1}}{L^{2}} \leq \inf _{N, f} \frac{\mathcal{E}_{L}^{J, N}(f, f)}{\operatorname{Var}_{L}^{J, N}(f)} \leq \frac{K_{2}}{L^{2}}
$$

where $K_{1}(\beta, J)$ and $K_{2}(\beta, J)$ are positive constants. We start proving the first of these inequalities.

The variance of every $f \in L^{2}\left(\nu_{L}^{J, N}\right)$ may be written as:

$$
\operatorname{Var}_{L}^{J, N}(f)=\mathbf{E}_{L}^{J, N}\left[\operatorname{Var}_{L}^{J, N}\left(f \mid \eta_{L}\right)\right]+\operatorname{Var}_{L}^{J, N}\left[\mathbf{E}_{L}^{J, N}\left(f \mid \eta_{L}\right)\right]
$$


Define $\tilde{J}=\left(J_{l}, 1\right)$, then $\operatorname{Var}_{L}^{J, N}\left(f \mid \eta_{L}\right)=\operatorname{Var}_{L-1}^{\tilde{J}, N-\eta_{L}}\left[f\left(\cdot \mid \eta_{L}\right)\right]$ so that by Theorem 2.1 the first term on the right hand side of (5.5) can be estimated as:

$$
\mathbf{E}_{L}^{J, N}\left[\operatorname{Var}_{L}^{J, N}\left(f \mid \eta_{L}\right)\right] \leq C_{1}\left(\beta, J_{l}\right) L^{2} \mathcal{E}_{L}^{J, N}(f, f)
$$

Now we may use the same technique used to prove (3.2) to prove the following Poincaré inequality:

$$
\operatorname{Var}_{L}^{J, N}(g) \leq C_{2}(\beta, J) \mathbf{E}_{L}^{J, N}\left[\left(\partial_{L}^{+} g\right)^{2}\right]
$$

for every $g$ local in $\eta_{L}$. In particular:

$$
\operatorname{Var}_{L}^{J, N}\left[\mathbf{E}_{L}^{J, N}\left(f \mid \eta_{L}\right)\right] \leq C_{2}(\beta, J) \mathbf{E}_{L}^{J, N}\left[\left(\partial_{L}^{+} \mathbf{E}_{L}^{J, N}\left(f \mid \eta_{L}\right)\right)^{2}\right]
$$

This relation and the analog of (4.11) give:

$$
\begin{aligned}
& \operatorname{Var}_{L}^{J, N}\left[\mathbf{E}_{L}^{J, N}\left(f \mid \eta_{L}\right)\right] \leq \\
\leq & C_{3}(\beta, J)\left\{\mathbf{E}_{L}^{J, N}\left[\left(\partial_{L, L-1} f\right)^{2}\right]+\mathbf{E}_{L}^{J, N}\left[\mathbf{E}_{L}^{J, N}\left(f, g_{L-1} \mid \eta_{L}\right)^{2}\right]\right\},
\end{aligned}
$$

for a bounded function $g_{L-1}$ local in $\eta_{L-1}$. By the Schwarz inequality and (5.6) we have:

$$
\operatorname{Var}_{L}^{J, N}\left[\mathbf{E}_{L}^{J, N}\left(f \mid \eta_{L}\right)\right] \leq C_{4}(\beta, J) L^{2} \mathcal{E}_{L}^{J, N}(f, f)
$$

This estimate and (5.5) prove the first inequality in (5.4).

The proof of the second inequality in (5.4) is a repetition of the proof of Proposition 5.1.

Proof of Corollary 2.3. This corollary may be proved in the same way we proved Corollary 2.2. The only difference is that in this case the constant $C_{2}$ depends on $N$.

\section{One Site Marginal}

In this section we will study the one site marginal of the measure $\nu_{L}^{\delta, N}$ with $\delta \in(0,1)$. In particular we will prove Proposition 3.1. Our main tools are the so called Cheeger inequality (see Lawler, Sokal (1988)) and the local limit theorem (see Petrov (1975), Chapter VII, Theorem 13). To keep notation simple we shall write $\mu_{k}(x) \equiv \nu_{L}^{\delta, N}\left(\eta_{k}=x\right)$.

We begin defining some auxiliary probability measures. For every real number $\lambda$ with $|\lambda|<\beta$ define on $\mathbb{Z}$ the probability measures:

$$
\nu_{j}^{\lambda}(\eta) \equiv \frac{e^{-\beta|\eta|+\lambda \eta}}{Z(\beta, \lambda)}, \quad \tilde{\nu}_{j}^{\lambda}(\eta) \equiv \frac{e^{-\beta \delta|\eta|+\lambda \eta}}{Z(\beta \delta, \lambda)}, \quad j \in \mathbb{N}
$$


where $Z(\beta, \lambda) \equiv \sum_{\eta} e^{-\beta|\eta|+\lambda \eta}>1$. Consider now the infinite product measures on $\Omega$ given by

$$
\nu^{\lambda} \equiv \bigotimes_{j=1}^{+\infty} \nu_{j}^{\lambda}, \quad \tilde{\nu} \equiv \tilde{\nu}_{1}^{0} \otimes \bigotimes_{j=2}^{+\infty} \nu_{j}^{0} ;
$$

it is clear that $\nu_{L}^{\delta, N}(\cdot)=\tilde{\nu}\left(\cdot \mid \eta_{1}+\cdot+\eta_{L}=N\right)$. Expectation with respect to $\nu^{\lambda}$ will be denoted by $\mathbf{E}^{\lambda}(\cdot)$ while $m(\lambda)$ and $\sigma^{2}(\lambda)$ stands respectively for the mean and the variance of $\eta_{1}$ with respect to $\nu^{\lambda}$. For $\lambda=0$ we will omit the superscript 0, e.g. $\mathbf{E}(\cdot)=\mathbf{E}^{0}(\cdot)$. The following lemma shows some simple properties of $\nu^{\lambda}$; the elementary proof is left to the reader:

Lemma 6.1. Define $p_{L}^{\lambda}(x) \equiv \nu^{\lambda}\left(\eta_{1}+\cdots+\eta_{L}=x\right)$. Then:

1. for every $L>0$ and $x \in \mathbb{Z}$ we have $p_{L}(x)=p_{L}(|x|)$;

2. $p_{L}(x) \leq p_{L}(y)$ for $|x| \geq|y|$;

3. for every fixed $\bar{\lambda} \in(0, \beta)$ and $k \in \mathbb{N}$ we have:

$$
\sup _{|\lambda| \leq \bar{\lambda}} \mathbf{E}^{\lambda}\left(\left|\eta_{1}\right|^{k}\right)<+\infty
$$

4. $m(\lambda)$ is an increasing, odd, $C^{\infty}$ function in $\lambda \in(-\beta, \beta)$, moreover:

$$
\lim _{\lambda \uparrow \beta} m(\lambda)=+\infty
$$

5. for every $\lambda \in \mathbb{R}$ with $|\lambda|<\beta$ and $x \in \mathbb{Z}$ we have:

$$
p_{L}^{\lambda}(x)=e^{\lambda x} p_{L}(x)\left[\frac{Z(\beta, 0)}{Z(\beta, \lambda)}\right]^{L} .
$$

In what follows will be crucial the following result that can be proved by direct computation:

Lemma 6.2. Suppose that $x \in \mathbb{Z}$ and $\delta \in(0,1)$. Then:

$$
e^{-\beta[\delta \operatorname{sign}(x)+1]} \leq \frac{\mu_{1}(x+1)}{\mu_{1}(x)} \leq e^{-\beta[\delta \operatorname{sign}(x)-1]} ;
$$

and for $k=2, \ldots, L$ :

$$
e^{-\beta[\operatorname{sign}(x)+\delta]} \leq \frac{\mu_{k}(x+1)}{\mu_{k}(x)} \leq e^{-\beta[\operatorname{sign}(x)-\delta]} .
$$

Proof of Proposition 3.1. For $k=1, \ldots, L$ define the generator $G_{k}$ on $L^{2}\left(\mathbb{Z}, \mu_{k}\right)$ as $\left(G_{k} f\right)(x)=\sum_{y} c_{k}(x, y)[f(y)-f(x)]$, where:

$$
c_{k}(x, y) \equiv \begin{cases}\left(\frac{\mu_{k}(y)}{\mu_{k}(x)}\right)^{\frac{1}{2}} & \text { if }|x-y|=1 \\ 0 & \text { otherwise. }\end{cases}
$$

The rates $c_{k}(x, y)$ are uniformly bounded. It is simple to check that $G_{k}$ is a self-adjoint, negative definite Markov generator. Thus in order to prove 
Proposition 3.1 we have to give a lower bound for its spectral gap $\lambda_{1}\left(G_{k}\right)$. The estimates we will prove are based on the so called Cheeger inequality, which in our case says (see Lawler, Sokal (1988), Theorem 2.5 and Remark 2 in that paper):

$$
\lambda_{1}\left(G_{k}\right) \geq \frac{q^{2}}{2 M}
$$

where:

$$
q \equiv \sup _{x \in \mathbb{Z}}\left[\left(\inf _{\substack{a>x \\ 0<\mu_{k}[a,+\infty)<1}} \frac{\mu_{k}(a)}{\mu_{k}[a,+\infty)}\right) \wedge\left(\inf _{\substack{b<x \\ 0<\mu_{k}(-\infty, b]<1}} \frac{\mu_{k}(b)}{\mu_{k}(-\infty, b]}\right)\right]
$$

and $M \equiv \sup _{x}\left\{c_{k}(x, x+1)+c_{k}(x, x-1)\right\}$. Because in our case $M$ is a bounded constant the proposition is proved if we can show that:

$$
\sup _{x \in \mathbb{Z}}\left[\left(\inf _{\substack{a>x \\ 0<\mu_{1}[a,+\infty)<1}} \frac{\mu_{1}(a)}{\mu_{1}[a,+\infty)}\right) \wedge\left(\inf _{\substack{b<x \\ 0<\mu_{1}(-\infty, b]<1}} \frac{\mu_{1}(b)}{\mu_{1}(-\infty, b]}\right)\right] \geq \frac{K_{1}}{\sqrt{L}},
$$

and that:

$$
\sup _{x \in \mathbb{Z}}\left[\left(\inf _{\substack{a>x \\ 0<\mu_{k}[a,+\infty)<1}} \frac{\mu_{k}(a)}{\mu_{k}[a,+\infty)}\right) \wedge\left(\inf _{\substack{b<x \\ 0<\mu_{k}(-\infty, b]<1}} \frac{\mu_{k}(b)}{\mu_{k}(-\infty, b]}\right)\right] \geq K_{2},
$$

for $k=2, \ldots, L$. The following "telescopic" representation is useful:

$$
\begin{aligned}
& \frac{\mu_{k}[a,+\infty)}{\mu_{k}(a)}=1+\sum_{x>a} \prod_{z=a}^{x-1} \frac{\mu_{k}(z+1)}{\mu_{k}(z)} ; \\
& \frac{\mu_{k}(-\infty, b]}{\mu_{k}(b)}=1+\sum_{x<b} \prod_{z=x}^{b-1} \frac{\mu_{k}(z)}{\mu_{k}(z+1)} .
\end{aligned}
$$

By this representation we have immediately (6.6). In fact by (6.3) for $k \geq 2$ and $z>0$ we know that $\frac{\mu_{k}(z+1)}{\mu_{k}(z)} \leq e^{-\beta(1-\delta)}<1$ and $\frac{\mu_{k}(z)}{\mu_{k}(z+1)} \leq e^{-\beta(1-\delta)}<1$ for $z<0$. Using (6.7) and (6.8) it is simple to prove respectively that for every $a>0$ the ratio $\frac{\mu_{k}(a)}{\mu_{k}[a,+\infty)}$ is bounded below by a positive constant and that the same is true for every $b>0$ for the ratio $\frac{\mu_{k}(b)}{\mu_{k}(-\infty, b]}$. This proves (6.6).

The proof of (6.5) is conceptually similar but the study of the ratio $\frac{\mu_{1}(z+1)}{\mu_{1}(z)}$ is not so simple. To understand why it is so, recall that by definition

$$
\frac{\mu_{1}(z+1)}{\mu_{1}(z)}=e^{-\beta \delta \operatorname{sign}(z)} \frac{p_{L-1}(N-z-1)}{p_{L-1}(N-z)} .
$$

For $|z-N| \leq \operatorname{cost} . \sqrt{L}$ by local limit theorem we have $\frac{p_{L-1}(N-z-1)}{p_{L}-1(N-z)} \simeq 1$. Thus in this case $\frac{\mu_{1}(z+1)}{\mu_{1}(z)} \simeq e^{-\beta \delta \operatorname{sign}(z)}$. This gives an "inward drift" as 
in the previous case. Problems arise when $|z-N| \gg \sqrt{L}$. Suppose that $0<N-z \leq \bar{\rho} L$ for a positive constant $\bar{\rho}$. By the "Cramér trick" (6.1) we have that:

$$
\frac{\mu_{1}(z+1)}{\mu_{1}(z)}=\frac{p_{L-1}^{\lambda}(N-z-1)}{p_{L-1}^{\lambda}(N-z)} e^{-\beta \delta \operatorname{sign}(z)+\lambda} .
$$

for every $\lambda \in(-\beta, \beta)$. Now let $\lambda_{z}$ be such that $m\left(\lambda_{z}\right)=\frac{N-z}{L-1}$, such a $\lambda_{z}$ exists by Lemma 6.1. By local limit theorem $\frac{p_{L}^{\lambda_{z}}(N-z-1)}{p_{L}^{\lambda_{z}}(N-z)} \simeq 1$ and in this case $\frac{\mu_{1}(z+1)}{\mu_{1}(z)} \simeq e^{-\beta \delta \operatorname{sign}(z)+\lambda_{z}}$. This equation implies that $\frac{\mu_{1}(z+1)}{\mu_{1}(z)}<1$ for $-\beta \delta \operatorname{sign}(z)+\lambda_{z}<0$. In particular if $z>0$ we have $-\beta \delta+\lambda_{z}<0$ if and only if $z>N-m(\beta \delta)(L-1)$. So we have to study $\lambda_{z}$ carefully.

Define $\bar{x} \equiv N-m(\beta \delta)(L-1)$. We claim that for every $\bar{\rho}>0$ there exists $K(\beta, \delta, \bar{\rho})$ and $\bar{L}(\beta, \delta, \bar{\rho})>0$ such that:

$$
\left(\inf _{\substack{a>\bar{x} \vee 0 \\ 0<\mu_{1}[a,+\infty)<1}} \frac{\mu_{1}(a)}{\mu_{1}[a,+\infty)}\right) \wedge\left(\inf _{\substack{b<\bar{x} \vee 0 \\ 0<\mu_{1}(-\infty, b]<1}} \frac{\mu_{1}(b)}{\mu_{1}(-\infty, b]}\right) \geq \frac{K}{\sqrt{L}}
$$

for every $L>\bar{L}$ and $N \in \mathbb{Z}$ such that $|N / L| \leq \bar{\rho}$. This relation and (6.4) gives (3.1). The proof of (6.9) is divided into several lemmas. Each lemma bounds the ratio $\frac{\mu_{1}(z+1)}{\mu_{1}(z)}$ in an interval where $\mu_{1}$ has a different qualitative aspect. Because $\nu_{L}^{\delta, N}\left(\eta_{k}=-x\right)=\nu_{L}^{\delta,-N}\left(\eta_{k}=x\right)$ it is not restrictive to suppose that $N \geq 0$.

Lemma 6.3. Suppose that $z \geq N \geq 0$. Then:

$$
\frac{\mu_{1}(z+1)}{\mu_{1}(z)} \leq e^{-\beta \delta}
$$

Proof. By part 5 of Lemma 6.1, we know that:

$$
\frac{\mu_{1}(z+1)}{\mu_{1}(z)}=e^{-\beta \delta} \frac{p_{L-1}(N-z-1)}{p_{L-1}(N-z)}
$$

but since $z \geq N$, part 2 of the same lemma implies that 1 is an upper bound for the ratio $\frac{p_{L-1}(N-z-1)}{p_{L-1}(N-z)}$.

Lemma 6.4. Suppose that $\varepsilon \in(0, m(\beta \delta)), \bar{\rho}>0$ and $N / L \in[0, \bar{\rho}]$. Then there exists $K_{1}(\beta, \delta, \varepsilon)$ and $K_{2}(\beta, \delta, \bar{\rho})>0$ such that:

$$
\frac{\mu_{1}(z+1)}{\mu_{1}(z)} \leq\left(1+\frac{K_{2}}{L}\right) e^{-K_{1}}
$$

for every $z \in \mathbb{Z}$ satisfying $[\bar{x}+\varepsilon(L-1)] \vee 0 \leq z \leq N$.

Proof. Let $\lambda_{z} \in(-\beta, \beta)$ be so that $\mathbf{E}^{\lambda_{z}}(\eta)=\frac{N-\bar{z}}{L-1}$. Lemma 6.1 yields:

$$
\frac{\mu_{1}(z+1)}{\mu_{1}(z)}=\frac{p_{L-1}^{\lambda_{z}}(N-z-1)}{p_{L-1}^{\lambda_{z}}(N-z)} e^{-\beta \delta(|z+1|-|z|)+\lambda_{z}} .
$$


Because $0 \leq z \leq N \leq \bar{\rho} L$, the ratio $\left|\frac{N-z}{L-1}\right|$ is bounded above by $2 \bar{\rho}$. This implies that $\left|\lambda_{z}\right| \leq m^{-1}(2 \bar{\rho})<\beta$ and local limit theorem can be used:

$$
\frac{p_{L-1}^{\lambda_{z}}(N-z-1)}{p_{L-1}^{\lambda_{z}}(N-z)}=\frac{\frac{1}{\sqrt{2 \pi}}+O\left(L^{-1}\right)}{\frac{1}{\sqrt{2 \pi}}+O\left(L^{-1}\right)}=1+O\left(L^{-1}\right),
$$

uniformly in $N$ and $z$ with $\left|\frac{N-z}{L-1}\right| \leq 2 \bar{\rho}$. Thus there exists $C_{1}(\beta, \delta, \bar{\rho})>0$ such that:

$$
\frac{\mu_{1}(z+1)}{\mu_{1}(z)} \leq\left(1+\frac{C_{1}}{L}\right) e^{-\beta \delta+\lambda_{z}}
$$

for $0 \leq z \leq N \leq \bar{\rho} L$. Now because $z \geq \bar{x}+\varepsilon(L-1)$, the ratio $\frac{N-z}{L-1}$ is bounded above by $\frac{N-\bar{x}}{L-1}-\varepsilon=m(\beta \delta)-\varepsilon$. This means that $\lambda_{z}=m^{-1}\left(\frac{N-z}{L-1}\right) \leq$ $m^{-1}(m(\beta \delta)-\varepsilon)$. This estimate together with $(6.12)$ gives $(6.11)$.

Lemma 6.5. Suppose that $\bar{\rho}>0$ and $N / L \in[0, \bar{\rho}]$. Then there exists $\bar{\varepsilon}(\beta, \delta, \bar{\rho})>0$ such that for every $\varepsilon \in(0, \bar{\varepsilon})$ it is possible to find $K_{1}(\beta, \delta, \varepsilon)$ and $K_{2}(\beta, \delta, \bar{\rho})>0$ so that:

$$
\frac{\mu_{1}(z+1)}{\mu_{1}(z)} \leq\left(1+\frac{K_{2}}{L}\right) e^{-K_{1}\left(\frac{z-\bar{x}}{L-1}\right)+o\left(L^{-1}\right)}
$$

for every $z \in \mathbb{Z}$ satisfying $\bar{x} \vee 0<z<[\bar{x}+\varepsilon(L-1)] \vee 0$.

Proof. Let $\varepsilon>0$ be small enough such that $0 \leq z \leq N$. By (6.12) we may write:

$$
\frac{\mu_{1}(z+1)}{\mu_{1}(z)} \leq\left(1+\frac{C_{1}}{L}\right) e^{-\beta \delta+\lambda_{z}},
$$

for a positive constant $C_{1}(\beta, \delta, \bar{\rho})$. Because

$$
\lambda_{z} \equiv m^{-1}\left(\frac{N-z}{L-1}\right)=m^{-1}\left(\frac{N-\bar{x}}{L-1}+\frac{\bar{x}-z}{L-1}\right)
$$

and $\frac{\bar{x}-z}{L-1} \in(-\varepsilon, 0)$, for $\varepsilon$ small enough we can expand $\lambda_{z}$ in Taylor series. This yields:

$$
\lambda_{z} \leq \beta \delta+C_{2}\left(\frac{\bar{x}-z}{L-1}\right)+o\left(L^{-1}\right)
$$

for a positive constant $C_{2}(\beta, \delta, \bar{\rho})$. This estimate together with $(6.14)$ completes the proof.

The following three lemmas can be proved in the same way we proved lemmas $6.3,6.4$ and 6.5 respectively. We omit the proof for brevity.

Lemma 6.6. Suppose that $z<0$. Then:

$$
\frac{\mu_{1}(z)}{\mu_{1}(z+1)} \leq e^{-\beta \delta}
$$

Lemma 6.7. Suppose that $\varepsilon \in(0, m(\beta \delta)), \bar{\rho}>0$ and $N / L \in[0, \bar{\rho}]$. Then there exists $K_{1}(\beta, \delta, \varepsilon)$ and $K_{2}(\beta, \delta, \bar{\rho})>0$ such that:

$$
\frac{\mu_{1}(z)}{\mu_{1}(z+1)} \leq\left(1+\frac{K_{2}}{L}\right) e^{-K_{1}}
$$


for every $z \in \mathbb{Z}$ satisfying $0 \leq z<[\bar{x}-\varepsilon(L-1)] \vee 0$.

Lemma 6.8. Suppose that $\bar{\rho}>0$ and $N / L \in[0, \bar{\rho}]$. Then there exists $\bar{\varepsilon}(\beta, \delta, \bar{\rho})>0$ such that for every $\varepsilon \in(0, \bar{\varepsilon})$ it is possible to find $K_{1}(\beta, \delta, \varepsilon)$ and $K_{2}(\beta, \delta, \bar{\rho})>0$ so that:

$$
\frac{\mu_{1}(z)}{\mu_{1}(z+1)} \leq\left(1+\frac{K_{2}}{L}\right) e^{-K_{1}\left(\frac{\bar{x}-z}{L-1}\right)+o\left(L^{-1}\right)}
$$

for every $z \in \mathbb{Z}$ satisfying $[\bar{x}-\varepsilon(L-1)] \vee 0 \leq z<\bar{x} \vee 0$.

We are now in a position to prove (6.5). Suppose that $a \geq N$, then from the estimate $(6.10)$ it follows that:

$$
\sum_{x>a} \prod_{z=a}^{x-1} \frac{\mu_{1}(z+1)}{\mu_{1}(z)} \leq 1+\sum_{x>a} e^{-\beta \delta(x-a)}=\frac{e^{-\beta \delta}}{1-e^{-\beta \delta}} .
$$

Suppose that $[\bar{x}+\varepsilon(L-1)] \vee 0 \leq a \leq N$, a simple calculation gives:

$$
\begin{aligned}
& \sum_{x>a} \prod_{z=a}^{x-1} \frac{\mu_{1}(z+1)}{\mu_{1}(z)}= \\
= & \sum_{x=a+1}^{N} \prod_{z=a}^{x-1} \frac{\mu_{1}(z+1)}{\mu_{1}(z)}+\left(\prod_{z=a}^{N-1} \frac{\mu_{1}(z+1)}{\mu_{1}(z)}\right) \sum_{x>N} \prod_{z=N}^{x-1} \frac{\mu_{1}(z+1)}{\mu_{1}(z)} ;
\end{aligned}
$$

because of (6.15) the last sum in this expression is bounded above by a positive constant so that:

$$
\sum_{x>a} \prod_{z=a}^{x-1} \frac{\mu_{1}(z+1)}{\mu_{1}(z)} \leq C_{1}(\beta, \delta) \sum_{x=a}^{N} \prod_{z=a}^{x-1} \frac{\mu_{1}(z+1)}{\mu_{1}(z)} .
$$

Now (6.11) can be used to bound the last term of this expression:

$$
\sum_{x=a}^{N} \prod_{z=a}^{x-1} \frac{\mu_{1}(z+1)}{\mu_{1}(z)} \leq\left(1+\frac{C_{3}}{L}\right)^{\bar{\rho} L} \sum_{x=0}^{+\infty} e^{-C_{2} x} \leq C_{4}(\beta, \delta, \varepsilon, \bar{\rho}) .
$$

In conclusion:

$$
\sum_{x>a} \prod_{z=a}^{x-1} \frac{\mu_{1}(z+1)}{\mu_{1}(z)} \leq C_{5}(\beta, \delta, \varepsilon, \bar{\rho})
$$

for every $a$ such that $[\bar{x}+\varepsilon(L-1)] \vee 0 \leq a \leq N$.

Suppose now that $\bar{x} \vee 0<a \leq \bar{x}+\varepsilon(L-1)$ and define $\bar{y} \equiv \bar{x}+\varepsilon(L-1)$. A simple calculation yields:

$$
\begin{aligned}
& \sum_{x>a} \prod_{z=a}^{x-1} \frac{\mu_{1}(z+1)}{\mu_{1}(z)}= \\
= & \sum_{x=a+1}^{\bar{y}} \prod_{z=a}^{x-1} \frac{\mu_{1}(z+1)}{\mu_{1}(z)}+\left(\prod_{z=a}^{\bar{y}-1} \frac{\mu_{1}(z+1)}{\mu_{1}(z)}\right) \sum_{x>\bar{y}} \prod_{z=\bar{y}}^{x-1} \frac{\mu_{1}(z+1)}{\mu_{1}(z)} .
\end{aligned}
$$


By equation (6.16) the last sum in this expression is bounded above by a positive constant and we have:

$$
\sum_{x>a} \prod_{z=a}^{x-1} \frac{\mu_{1}(z+1)}{\mu_{1}(z)} \leq C_{6}(\beta, \delta, \varepsilon, \bar{\rho}) \sum_{x=a+1}^{\bar{y}} \prod_{z=a}^{x-1} \frac{\mu_{1}(z+1)}{\mu_{1}(z)}
$$

The estimate (6.13) may be used to bound last term in (6.17):

$$
\begin{aligned}
& \sum_{x=a}^{\bar{y}} \prod_{z=a}^{x-1} \frac{\mu_{1}(z+1)}{\mu_{1}(z)} \leq \sum_{x=a}^{\bar{y}} \prod_{z=a}^{x-1}\left(1+\frac{C_{8}}{L}\right) e^{-C_{7}\left(\frac{z-\bar{x}}{L-1}\right)+o\left(L^{-1}\right)} \leq \\
\leq & \left(1+\frac{C_{8}}{L}\right)^{N} e^{o\left(L^{-1}\right) \bar{y}} \sum_{x=a}^{\bar{y}} e^{-\frac{C_{7}}{L-1} \sum_{z=a}^{x-1}(z-\bar{x})} \leq C_{9} \sum_{x=a}^{\bar{y}} e^{-\frac{C_{7}}{L-1} \sum_{z=a}^{x-1}(z-\bar{x})}
\end{aligned}
$$

where $C_{7}(\beta, \delta, \varepsilon)$ and $C_{9}(\beta, \delta, \bar{\rho})$ are positive constants. A few calculations show that $\sum_{z=a}^{x-1}(z-\bar{x}) \geq \frac{1}{2}\left[(x-a)-\frac{1}{2}\right]^{2}-\frac{1}{8}$ for every $a \in(\bar{x}, x)$. This estimate and some elementary inequalities yield:

$$
\begin{aligned}
& \sum_{x=a}^{\bar{y}} e^{-\frac{C_{7}}{L-1} \sum_{z=a}^{x-1}(z-\bar{x})} \leq C_{10} \sum_{x=0}^{+\infty} e^{-\frac{C_{7}}{2(L-1)}\left(x-\frac{1}{2}\right)^{2}} \leq \\
\leq & C_{11} \int_{0}^{+\infty} e^{-\frac{C_{7}}{2(L-1)}\left(x-\frac{1}{2}\right)^{2}} d x \leq C_{12}(\beta, \delta, \varepsilon, \bar{\rho}) \sqrt{L} .
\end{aligned}
$$

This inequality together with (6.17) and (6.18) shows that

$$
\sum_{x>a} \prod_{z=a}^{x-1} \frac{\mu_{1}(z+1)}{\mu_{1}(z)} \leq C_{13} \sqrt{L}
$$

for every a such that $\bar{x}<a \leq \bar{x} \vee 0+\varepsilon(L-1)$, where $C_{13}(\beta, \delta, \varepsilon, \bar{\rho})$ is a positive constant.

By $(6.7),(6.15),(6.16)$ and $(6.19)$ we have:

$$
\inf _{\substack{a>\bar{x} \vee 0 \\ 0<\mu_{1}[a,+\infty)<1}} \frac{\mu_{1}(a)}{\mu_{1}[a,+\infty)}>\frac{C}{\sqrt{L}}
$$

for a positive constant $C(\beta, \delta, \varepsilon, \bar{\rho})$ and $L$ large enough.

In order to complete the proof of $(6.9)$ we need to prove that:

$$
\inf _{\substack{b<\bar{x} \vee 0 \\ 0<\mu_{1}(-\infty, b]<1}} \frac{\mu_{1}(b)}{\mu_{1}(-\infty, b]}>\frac{C}{\sqrt{L}} .
$$

The proof of this inequality is omitted since it follows closely the proof of (6.20). This concludes the proof of Proposition 3.1.

The last part of this section is dedicated to the proofs of some large deviation results for $\mu_{1}$. More precisely we will prove Lemma 3.2 and Lemma 3.3. 
Proof of Lemma 3.2. Because $\nu_{L}^{\delta, N}\left(\eta_{1}<0\right)=\nu_{L}^{\delta,-N}\left(\eta_{1}>0\right)$, we will prove only (3.3).

Define $\rho \equiv N / L$ and $\lambda_{\rho}$ such that $m\left(\lambda_{\rho}\right)=\rho$ (see Lemma 6.1). A simple calculation shows that:

$$
\begin{aligned}
& \nu_{L}^{\delta, N}\left(\eta_{1}<0\right)=\sum_{x<0} \frac{\tilde{\nu}\left(\eta_{1}=x, \bar{\eta}=N\right)}{\tilde{\nu}(\bar{\eta}=N)}=\sum_{x<0} \frac{e^{-\beta \delta|x|} p_{L-1}(N-x)}{Z(\beta \delta, 0) \tilde{\nu}(\bar{\eta}=N)} \leq \\
\leq & \frac{e^{\beta \delta N}\left[\mathbf{E}\left(e^{\lambda_{\rho} \eta}\right)\right]^{L-1}}{p_{L-1}(0)} \sum_{x>0} e^{-\beta \delta x} p_{L-1}^{\lambda_{\rho}}(N+x) e^{-\lambda_{\rho}(N+x)} \leq \\
\leq & \frac{e^{\left(\beta \delta-\lambda_{\rho}\right) N}\left[\mathbf{E}\left(e^{\lambda_{\rho} \eta}\right)\right]^{L-1}}{p_{L-1}(0)} \sum_{x>0} e^{-\beta \delta x}=\frac{e^{-\beta \delta}}{1-e^{-\beta \delta}} \frac{e^{\left(\beta \delta-\lambda_{\rho}\right) N} \mathbf{E}\left(e^{\lambda_{\rho} \eta}\right)^{L-1}}{p_{L-1}(0)} \leq \\
\leq & C_{1}(\beta, \delta) \sqrt{L} e^{\left(\beta \delta-\lambda_{\rho}\right) N} \mathbf{E}\left(e^{\lambda_{\rho} \eta}\right)^{L-1}
\end{aligned}
$$

where we used the trivial estimate $\tilde{\nu}\left(\eta_{1}+\cdots+\eta_{L}=N\right) \geq \tilde{\nu}\left(\eta_{1}=N\right) p_{L-1}(0)$ and the local limit theorem. In order to prove (3.3) we have to show that there exists $C_{1}(\beta, \delta)$ and $\bar{\rho}(\beta, \delta)>0$ such that $e^{\rho\left(\beta \delta-\lambda_{\rho}\right)} \mathbf{E}\left(e^{\lambda_{\rho} \eta}\right)<e^{-C_{1}}$ for $\rho>\bar{\rho}(\beta, \delta)$. Direct calculation yields:

$$
\mathbf{E}\left(e^{\lambda \eta}\right)=\frac{1}{Z(\beta, 0)}\left[\frac{e^{-(\beta+\lambda)}}{1-e^{-(\beta+\lambda)}}+\frac{1}{1-e^{\lambda-\beta}}\right] \leq \frac{C_{3}(\beta)}{1-e^{\lambda-\beta}},
$$

for $\lambda \in(0, \beta)$ so that

$$
e^{\rho\left(\beta \delta-\lambda_{\rho}\right)} \mathbf{E}\left(e^{\lambda_{\rho} \eta}\right) \leq C_{3}(\beta) \frac{e^{\rho\left(\beta \delta-\lambda_{\rho}\right)}}{1-e^{\lambda_{\rho}-\beta}} .
$$

We claim that the last factor in this estimate can be made smaller than 1 taking $\rho$ large enough. In fact a simple calculation permits us to write explicitly the "Cramér transform":

$$
\lambda_{\rho}=\log \left[\frac{\rho\left(e^{\beta}+e^{-\beta}\right)+\sqrt{\rho^{2}\left(e^{\beta}-e^{-\beta}\right)^{2}+4}}{2(\rho+1)}\right],
$$

and it is easy to check that $1-e^{\lambda_{\rho}-\beta}=O\left(\rho^{-1}\right)$ for $\rho \rightarrow+\infty$.

Proof of Lemma 3.3. Notice that $\nu_{L}^{\delta, N}\left(\left|\eta_{1}\right|>M L\right)=\nu_{L}^{\delta, N}\left(\eta_{1}>M L\right)+$ $\nu_{L}^{\delta,-N}\left(\eta_{1}>M L\right)$, so we need to estimate only $\nu_{L}^{\delta, N}\left(\eta_{1}>M L\right)$.

An elementary calculation yields:

$$
\begin{aligned}
& \nu_{L}^{\delta, N}\left(\eta_{1}>M L\right)=\sum_{x>M L} \nu_{L}^{\delta, N}\left(\eta_{1}=x\right)= \\
= & \frac{1}{\tilde{\nu}(\bar{\eta}=N) Z(\beta \delta, 0)} \sum_{x>M L} e^{-\beta \delta x} p_{L-1}(N-x) \leq \\
\leq & \frac{e^{\beta \delta N}}{p_{L-1}(0)} \sum_{x>M L} e^{-\beta \delta x} p_{L-1}(N-x) \leq \\
\leq & \frac{e^{\beta \delta N}}{p_{L-1}(0)} \sum_{x>M L} e^{-\beta \delta x} \leq C_{1}(\beta, \delta) \sqrt{L} \sum_{x>M L} e^{-\beta \delta x} \leq \\
\leq & C_{2}(\beta, \delta) \sqrt{L} e^{-\beta \delta(M L-N)} \leq C_{2}(\beta, \delta) \sqrt{L} e^{-\beta \delta(M-\bar{\rho}) L}
\end{aligned}
$$

where we used the trivial estimate: $\tilde{\nu}\left(\eta_{1}+\cdots \eta_{L}=N\right) \geq \tilde{\nu}\left(\eta_{1}=N\right) p_{L-1}(0)$ and the local limit theorem. 


\section{Two Block Estimate}

In this section we will prove Proposition 3.4. This is a generalization of a similar result obtained by H. T. Yau (see Yau (1994)) in the simpler context of bounded random variables. Our main tool is again local limit theorem (see Petrov (1975), Chapter VII, Theorem 13). Throughout this section we will assume $\delta=1$ and $|N / L| \leq \bar{\rho}<+\infty$. We will use notation and results from Section 6 .

The proof of Proposition 3.4 requires some preliminary results. Let $k \leq L$ be a positive fixed integer. Consider the partition $\mathcal{A} \equiv\left\{\alpha_{1}, \ldots, \alpha_{L / k}\right\}$ of $[1, L] \cap \mathbb{N}$, where $\alpha_{i} \equiv[1+(i-1) k, i k] \cap[1, L] \cap \mathbb{N}$. Let $\left\{g_{\alpha}\right\}_{\alpha \in \mathcal{A}}$ be a family of random variables indexed on $\mathcal{A}$, we denote by $\mathrm{Av}_{\alpha} g_{\alpha}$ the arithmetic mean of the family $\operatorname{Av}_{\alpha} g_{\alpha} \equiv \frac{k}{L} \sum_{i=1}^{L / k} g_{\alpha_{i}}$.

Lemma 7.1. Suppose that $F: \mathbb{R}^{k} \rightarrow \mathbb{R}$ is such that:

$$
\left|F\left(x_{1}, \ldots, x_{k}\right)\right| \leq K_{1}+\frac{K_{2}}{k} \sum_{i=1}^{k}\left|x_{i}\right|
$$

for two constants $K_{1}$ and $K_{2}>0$. For any $\alpha \in \mathcal{A}$ define $F_{\alpha}(\eta) \equiv F\left(\eta_{\alpha}\right)$ and suppose that $\mathbf{E}_{L}^{N}\left(F_{\alpha}\right)=0$. Then there exists $\bar{\theta}(\beta, \bar{\rho}, F)>0$ so that:

$$
\mathbf{E}_{L}^{N}\left[\left(\mathrm{Av}_{\alpha} F_{\alpha}\right)^{2}\right] \leq \frac{4}{\theta^{2}} \log \mathbf{E}_{L}^{N}\left(e^{\theta \mathrm{A} \mathbf{v}_{\alpha} F_{\alpha}}\right)
$$

for any $|\theta| \leq \bar{\theta}$.

Proof. A formal expansion of the exponential function yields:

$$
\mathbf{E}_{L}^{N}\left(e^{\theta \mathrm{A} \mathrm{v}_{\alpha} F_{\alpha}}\right)=1+\frac{\theta^{2}}{2} \mathbf{E}_{L}^{N}\left[\left(\mathrm{Av}_{\alpha} F_{\alpha}\right)^{2}\right]+o\left(\theta^{2}\right)
$$

from which (7.2) follows for $\theta$ small enough. Thus the lemma is proved if we can show that the expansion (7.3) is correct. It is elementary to prove that (7.3) is true if there exists a positive constant $K(\beta, \bar{\rho}, F)$ such that $\mathbf{E}_{L}^{N}\left(\left|\mathrm{Av}_{\alpha} F_{\alpha}\right|^{n}\right) \leq K^{n} n$ !. Because of condition (7.1) this is true if we can show that

$$
\mathbf{E}_{L}^{N}\left(\left|\eta_{1}\right|^{n}\right) \leq C_{1}^{n} n !
$$

We are going to prove this estimate.

Let $\lambda_{\rho}$ be such that $\mathbf{E}^{\lambda_{\rho}}(\eta)=\rho \equiv N / L$ (see Lemma 6.1 ). By part 5 in Lemma 6.1 we can write:

$$
\begin{aligned}
& \quad \mathbf{E}_{L}^{N}\left(\left|\eta_{1}\right|^{n}\right)=\sum_{|x| \leq \sqrt{L}}|x|^{n} \nu\left(\eta_{1}=x\right) \frac{p_{L-1}^{\lambda_{\rho}}(N-x) e^{\lambda_{\rho} x}}{p_{L}^{\lambda_{\rho}}(N) Z\left(\beta, \lambda_{\rho}\right)}+ \\
& +\sum_{|x|>\sqrt{L}}|x|^{n} \nu\left(\eta_{1}=x\right) \frac{p_{L-1}^{\lambda_{\rho}}(N-x) e^{\lambda_{\rho} x}}{p_{L}^{\lambda_{\rho}}(N) Z\left(\beta, \lambda_{\rho}\right)} .
\end{aligned}
$$


Local limit theorem may be invoked to claim that the ratio $\frac{p_{L}^{\lambda_{\rho}}-1}{\left.p_{L}^{\lambda_{\rho}}(N)-x\right)}$ is bounded by a positive constant depending only on $\bar{\rho}$ for $|x| \leq \sqrt{L}$. This implies that the first term on the right hand side of (7.5) is bounded above by $C_{2}(\beta, \bar{\rho}) \mathbf{E}\left(\left|\eta_{1}\right|^{n} e^{\lambda_{\rho} \eta_{1}}\right)$.

Again local limit theorem can be used to claim that $p_{L}^{\lambda_{\rho}}(N)=O\left(L^{-1 / 2}\right)$, thus there exists a positive constant $C_{3}(\beta, \bar{\rho})$ such that the second term on the right hand side of (7.5) is bounded above by:

$$
C_{3} \sqrt{L} \sum_{|x|>\sqrt{L}}|x|^{n} \nu\left(\eta_{1}=x\right) e^{\lambda_{\rho} x} \leq C_{3} \sqrt{L} e^{-t \sqrt{L}} \sum_{|x|>\sqrt{L}}|x|^{n} \nu\left(\eta_{1}=x\right) e^{\left(\lambda_{\rho}+t\right) x}
$$

where $t>0$ is such that $\left|t+\lambda_{\rho}\right|<\beta$ for any $|\rho| \leq \bar{\rho}$. If $L$ is large enough the term on right hand side of this inequality is bounded above by $C_{4}(\beta, \bar{\rho}) \mathbf{E}\left[\left|\eta_{1}\right|^{n} e^{\left(\lambda_{\rho}+t\right) \eta_{1}}\right]$.

In conclusion $\mathbf{E}_{L}^{N}\left(\left|\eta_{1}\right|^{n}\right) \leq C_{5} \mathbf{E}\left(\left|\eta_{1}\right|^{n} e^{\bar{\lambda} \eta_{1}}\right)$ for a fixed $\bar{\lambda} \in(-\beta, \beta)$. In order to prove (7.4) we have to show that for any $\lambda \in(-\beta, \beta)$ there exists $C(\beta, \lambda)>0$ such that $\mathbf{E}\left(\left|\eta_{1}\right|^{n} e^{\lambda \eta_{1}}\right) \leq C(\beta, \lambda)^{n} n$ !. This fact may be proved by direct calculation.

Next lemma is useful to calculate some probabilistic quantities related with the measure $\nu_{L}^{N}$. If $f, g, h$ are random variables we define $\mathbf{E}^{\lambda}(f, g, h)$ as

$$
\mathbf{E}^{\lambda}(f, g, h) \equiv \mathbf{E}^{\lambda}\left[\left(f-\mathbf{E}^{\lambda}(f)\right)\left(g-\mathbf{E}^{\lambda}(g)\right)\left(h-\mathbf{E}^{\lambda}(h)\right)\right] .
$$

Proposition 7.2. In the same setting as in Lemma 3.4, except that not necessarily $\mathbf{E}_{L}^{N}\left(F_{\alpha}\right)=0$, there exists $\bar{\theta}(\beta, \bar{\rho}, F)>0$ such that:

$$
\frac{1}{\theta} \log \mathbf{E}_{L}^{N}\left(e^{\theta \mathrm{A} \mathbf{v}_{\alpha} F_{\alpha}}\right)=\mathbf{E}^{\lambda_{\rho}}\left(F_{\alpha}\right)+\frac{k}{L}\left[G_{1}\left(F_{\alpha}, \rho\right)+\theta G_{2}\left(F_{\alpha}, \rho\right)\right]+o\left(L^{-1}\right)
$$

for any $|\theta| \leq \bar{\theta}$. Here $\lambda_{\rho}$ is such that $\mathbf{E}^{\lambda_{\rho}}\left(\eta_{1}\right)=\rho \equiv N / L$ and:

$$
\begin{aligned}
& G_{1}\left(F_{\alpha}, \rho\right) \equiv \frac{1}{2}\left[\frac{\mathbf{E}^{\lambda_{\rho}}\left(\bar{\eta}_{\alpha}, \bar{\eta}_{\alpha}, \bar{\eta}_{\alpha}\right) \mathbf{E}^{\lambda_{\rho}}\left(F_{\alpha}, \bar{\eta}_{\alpha}\right)}{\operatorname{Var}^{\lambda_{\rho}}\left(\bar{\eta}_{\alpha}\right)^{2}}-\frac{\mathbf{E}^{\lambda_{\rho}}\left(\bar{\eta}_{\alpha}, \bar{\eta}_{\alpha}, F_{\alpha}\right)}{\operatorname{Var}^{\lambda_{\rho}}\left(\bar{\eta}_{\alpha}\right)}\right] \\
& G_{2}\left(F_{\alpha}, \rho\right) \equiv \frac{1}{2}\left[\mathbf{E}^{\lambda_{\rho}}\left(F_{\alpha}^{2}\right)-\frac{\mathbf{E}^{\lambda_{\rho}}\left(F_{\alpha}, \bar{\eta}_{\alpha}\right)^{2}}{\operatorname{Var}^{\lambda_{\rho}}\left(\bar{\eta}_{\alpha}\right)}\right] .
\end{aligned}
$$

Proof. Notice that there exists $\bar{\varepsilon}(\beta, \bar{\rho}, F)>0$ and $\gamma=\gamma(\varepsilon)$ such that:

$$
\frac{\mathbf{E}^{\lambda_{\rho}}\left[\bar{\eta}_{\alpha} e^{\varepsilon F_{\alpha}+\gamma(\varepsilon) \bar{\eta}_{\alpha}}\right]}{\mathbf{E}^{\lambda_{\rho}}\left[e^{\varepsilon F_{\alpha}+\gamma(\varepsilon) \bar{\eta}_{\alpha}}\right]}=k \rho
$$

for any $|\varepsilon| \leq \bar{\varepsilon}$. In fact (7.9) is equivalent to:

$$
\mathbf{E}^{\lambda_{\rho}}\left[\left(\bar{\eta}_{\alpha}-k \rho\right) e^{\varepsilon F_{\alpha}+\gamma\left(\bar{\eta}_{\alpha}-k \rho\right)}\right]=0
$$


by the implicit function theorem there exists $\bar{\varepsilon}(\beta, \bar{\rho}, F)>0$ and a function $\gamma \in C^{1}(-\bar{\varepsilon}, \bar{\varepsilon})$ satisfying (7.9). The same theorem implies that:

$$
\gamma(0)=0, \quad \gamma^{\prime}(0)=-\frac{\mathbf{E}^{\lambda_{\rho}}\left(F_{\alpha}, \bar{\eta}_{\alpha}\right)}{\operatorname{Var}^{\lambda_{\rho}}\left(\bar{\eta}_{\alpha}\right)} .
$$

Fix some $|\varepsilon|<\bar{\varepsilon}(\beta, \bar{\rho}, F)$ and define on $\Omega$ the probability measure $\nu^{\lambda_{\rho}, \varepsilon} \equiv$ $\bigotimes_{\alpha} \nu_{\alpha}^{\lambda_{\rho}, \varepsilon}$, where

$$
\nu_{\alpha}^{\lambda_{\rho}, \varepsilon}\left(\eta_{\alpha}\right) \equiv \frac{e^{\varepsilon F_{\alpha}+\gamma(\varepsilon) \bar{\eta}_{\alpha}}}{\mathbf{E}^{\lambda_{\rho}}\left[e^{\varepsilon F_{\alpha}+\gamma(\varepsilon) \bar{\eta}_{\alpha}}\right]}
$$

With respect to this measure $\left\{\bar{\eta}_{\alpha}\right\}_{\alpha \in \mathcal{A}}$ are independent identically distributed random variables with expected value $\mathbf{E}^{\lambda_{\rho}, \varepsilon}\left(\bar{\eta}_{\alpha}\right)=k \rho$ and variance:

$$
\sigma^{2}\left(\lambda_{\rho}, \varepsilon\right)=\frac{\mathbf{E}^{\lambda_{\rho}}\left[\left(\bar{\eta}_{\alpha}-k \rho\right)^{2} e^{\varepsilon F_{\alpha}+\gamma \bar{\eta}_{\alpha}}\right]}{\mathbf{E}^{\lambda_{\rho}}\left(e^{\varepsilon F_{\alpha}+\gamma \bar{\eta}_{\alpha}}\right)}=\frac{\mathbf{E}^{\lambda_{\rho}}\left[\left(\bar{\eta}_{\alpha}-k \rho\right)^{2} e^{\varepsilon F_{\alpha}+\gamma\left(\bar{\eta}_{\alpha}-k \rho\right)}\right]}{\mathbf{E}^{\lambda_{\rho}}\left[e^{\varepsilon F_{\alpha}+\gamma\left(\bar{\eta}_{\alpha}-k \rho\right)}\right]} .
$$

Define $\bar{F} \equiv F_{\alpha_{1}}+\cdots+F_{\alpha_{L / k}}$ and notice that:

$$
\begin{aligned}
& \mathbf{E}_{L}^{N}\left(e^{\varepsilon \bar{F}}\right)=\frac{\mathbf{E}^{\lambda_{\rho}}\left[e^{\varepsilon \bar{F}} \mathbf{1}(\bar{\eta}=N)\right]}{\mathbf{E}^{\lambda_{\rho}}[\mathbf{1}(\bar{\eta}=N)]}=\frac{\nu^{\lambda_{\rho}, \varepsilon}(\bar{\eta}=N)}{\nu^{\lambda_{\rho}, 0}(\bar{\eta}=N)} \mathbf{E}^{\lambda_{\rho}}\left[e^{\varepsilon \bar{F}+\gamma(\bar{\eta}-N)]=}\right. \\
= & \sqrt{\frac{\sigma^{2}\left(\lambda_{\rho}, 0\right)}{\sigma^{2}\left(\lambda_{\rho}, \varepsilon\right)}} \frac{\frac{1}{\sqrt{2 \pi}}+\frac{k}{L} q_{2}^{\lambda_{\rho}, \varepsilon, k}(0)+o\left(L^{-1}\right)}{\frac{1}{\sqrt{2 \pi}}+\frac{k}{L} q_{2}^{\lambda_{\rho}, 0, k}(0)+o\left(L^{-1}\right)} \mathbf{E}^{\lambda_{\rho}}\left[e^{\varepsilon F_{\alpha}+\gamma\left(\bar{\eta}_{\alpha}-k \rho\right)}\right]^{L / k}
\end{aligned}
$$

where we used local limit theorem in the last line ( $q_{2}$ is a quadratic polynomial defined in Petrov (1975), Chapter VII, Theorem 13). Substituting $\varepsilon=\frac{k}{L} \theta$ the previous formula may be rewritten as:

$$
\begin{aligned}
& \mathbf{E}_{L}^{N}\left(e^{\theta \mathrm{Av}_{\alpha} F_{\alpha}}\right)=\sqrt{\frac{\sigma^{2}\left(\lambda_{\rho}, 0\right)}{\sigma^{2}\left(\lambda_{\rho}, \frac{\theta k}{L}\right)}} \frac{\frac{1}{\sqrt{2 \pi}}+\frac{k}{L} q_{2}^{\lambda_{\rho}, \frac{\theta k}{L}, k}(0)+o\left(L^{-1}\right)}{\frac{1}{\sqrt{2 \pi}}+\frac{k}{L} q_{2}^{\lambda_{\rho}, 0, k}(0)+o\left(L^{-1}\right)} \times \\
\times & \mathbf{E}^{\lambda_{\rho}}\left[e^{\theta \mathrm{Av}_{\alpha} F_{\alpha}+\gamma\left(\frac{\theta k}{L}\right)\left(\bar{\eta}_{\alpha}-k \rho\right)}\right]^{L / k} .
\end{aligned}
$$

Taylor's expansion, (7.10), and a few calculations yields:

$$
\begin{aligned}
& \frac{\frac{1}{\sqrt{2 \pi}}+\frac{k}{L} q_{2}^{\lambda_{\rho}, \frac{\theta k}{L}, k}(0)+o\left(L^{-1}\right)}{\frac{1}{\sqrt{2 \pi}}+\frac{k}{L} q_{2}^{\lambda_{\rho}, 0, k}(0)+o\left(L^{-1}\right)}=1+o\left(L^{-1}\right) \\
& \mathbf{E}^{\lambda_{\rho}}\left[e^{\theta A v_{\alpha} F_{\alpha}+\gamma\left(\frac{\theta k}{L}\right)\left(\bar{\eta}_{\alpha}-k \rho\right)}\right]^{L / k}= \\
& =1+\theta \mathbf{E}^{\lambda_{\rho}}\left(F_{\alpha}\right)+\frac{\theta^{2} k}{L} G_{2}\left(F_{\alpha}, \rho\right)+o\left(L^{-1}\right) \\
& \sqrt{\frac{\sigma^{2}\left(\lambda_{\rho}, 0\right)}{\sigma^{2}\left(\lambda_{\rho}, \frac{\theta k}{L}\right)}}=1+G_{1}\left(F_{\alpha}, \rho\right) \frac{\theta k}{L}+o\left(L^{-1}\right) .
\end{aligned}
$$


Now using (7.11) and (7.12), it is elementary to prove (7.6).

As a consequence of Proposition 7.2 we have the following results that can be read as "equivalence of ensembles".

Corollary 7.3. In the same setting as in Proposition 7.2 we have:

$$
\mathbf{E}_{L}^{N}\left(F_{\alpha}\right)=\mathbf{E}^{\lambda_{\rho}}\left(F_{\alpha}\right)+\frac{k}{L} G_{1}\left(F_{\alpha}, \rho\right)+o\left(L^{-1}\right) .
$$

Proof. For every $\theta \in(0, \bar{\theta})$ the Jensen inequality yields

$$
\mathbf{E}_{L}^{N}\left(F_{\alpha}\right)=\mathbf{E}_{L}^{N}\left(\mathrm{Av}_{\alpha} F_{\alpha}\right)=\frac{1}{\theta} \mathbf{E}_{L}^{N}\left(\log e^{\theta \mathrm{A} \mathbf{v}_{\alpha} F_{\alpha}}\right) \leq \frac{1}{\theta} \log \mathbf{E}_{L}^{N}\left(e^{\theta \mathrm{A} \mathbf{v}_{\alpha} F_{\alpha}}\right)
$$

and:

$$
\mathbf{E}_{L}^{N}\left(F_{\alpha}\right)=-\frac{1}{\theta} \mathbf{E}_{L}^{N}\left(\log e^{-\theta A \mathbf{v}_{\alpha} F_{\alpha}}\right) \geq-\frac{1}{\theta} \log \mathbf{E}_{L}^{N}\left(e^{-\theta A \mathbf{v}_{\alpha} F_{\alpha}}\right)
$$

These estimates and (7.6) give:

$$
\begin{aligned}
& -\frac{k \theta}{L} G_{2}\left(F_{\alpha}, \rho\right)+o\left(L^{-1}\right) \leq \mathbf{E}_{L}^{N}\left(F_{\alpha}\right)-\mathbf{E}^{\lambda_{\rho}}\left(F_{\alpha}\right)-\frac{k}{L} G_{1}\left(F_{\alpha}, \rho\right) \leq \\
& \leq \frac{k \theta}{L} G_{2}\left(F_{\alpha}, \rho\right)+o\left(L^{-1}\right) .
\end{aligned}
$$

Taking the limit for $\theta \downarrow 0$ we have (7.13).

Corollary 7.4. In the same setting as in Proposition 7.2 we have:

$$
\operatorname{Var}_{L}^{N}\left(\operatorname{Av}_{\alpha} F_{\alpha}\right) \leq 4 \frac{k}{L} \tilde{G}_{2}\left(F_{\alpha}, \rho\right)+o\left(L^{-1}\right),
$$

where:

$$
\tilde{G}_{2}\left(F_{\alpha}, \rho\right) \equiv \frac{1}{2}\left[\operatorname{Var}^{\lambda_{\rho}}\left(F_{\alpha}\right)-\frac{\mathbf{E}^{\lambda_{\rho}}\left(F_{\alpha}, \bar{\eta}_{\alpha}\right)^{2}}{\operatorname{Var}^{\lambda_{\rho}}\left(\bar{\eta}_{\alpha}\right)}\right]
$$

Proof. We can suppose $\mathbf{E}_{L}^{N}\left(F_{\alpha}\right)=0$. Lemma 7.1 and Proposition 7.2 give:

$$
\begin{aligned}
& \mathbf{E}_{L}^{N}\left[\left(\mathrm{Av}_{\alpha} F_{\alpha}\right)^{2}\right] \leq \frac{4}{(\bar{\theta} / 2)^{2}} \log \mathbf{E}_{L}^{N}\left(e^{\frac{\bar{\theta}}{2} \mathrm{~A} \mathbf{v}_{\alpha} F_{\alpha}}\right)= \\
= & \frac{4}{(\bar{\theta} / 2)}\left\{\mathbf{E}^{\lambda_{\rho}}\left(F_{\alpha}\right)+\frac{k}{L}\left[G_{1}\left(F_{\alpha}, \rho\right)+\frac{\bar{\theta}}{2} G_{2}\left(F_{\alpha}, \rho\right)\right]\right\}+o\left(L^{-1}\right) .
\end{aligned}
$$

But by Corollary 7.3 we know that $\mathbf{E}^{\lambda_{\rho}}\left(F_{\alpha}\right)=-\frac{k}{L} G_{1}\left(F_{\alpha}, \rho\right)+o\left(L^{-1}\right)$, thus:

$$
\mathbf{E}_{L}^{N}\left[\left(\mathrm{Av}_{\alpha} F_{\alpha}\right)^{2}\right] \leq 4 \frac{k}{L} G_{2}\left(F_{\alpha}, \rho\right)+o\left(L^{-1}\right) .
$$

Notice that

$$
G_{2}\left(F_{\alpha}, \rho\right)-\tilde{G}_{2}\left(F_{\alpha}, \rho\right)=\mathbf{E}^{\lambda_{\rho}}\left(F_{\alpha}\right)^{2}+o\left(L^{-1}\right)
$$


and that $\mathbf{E}^{\lambda_{\rho}}\left(F_{\alpha}\right)^{2}=o\left(L^{-1}\right)$. This fact together with (7.15) implies (7.14).

We are finally in a position to prove Proposition 3.4.

Proof of Proposition 3.4. The proof is divided into several steps for purpose of clarity.

STEP 1 . For every $\varepsilon>0$ there exists $\bar{n}(\beta, \bar{\rho})$ and $\bar{k}(\beta, \varepsilon, \bar{\rho}, F)>0$ such that for any $k>\bar{k}$ it is possible to find $\bar{L}(\beta, \bar{\rho}, \varepsilon, k)>0$ so that:

$$
\mathbf{E}_{L}^{N}\left(f, \mathrm{Av}_{j} h_{j}\right)^{2} \leq \frac{\varepsilon}{L} \operatorname{Var}_{L}^{N}(f)+2 \mathbf{E}_{L}^{N}\left[f, \mathrm{Av}_{\alpha} \mathbf{1}\left(\left|\bar{\eta}_{\alpha}\right| \leq \bar{n} k\right) \mathrm{Av}_{j \in \alpha} h_{j}\right]^{2}
$$

for every $L>\bar{L}$.

Proof of Step 1. A few calculations yields:

$$
\begin{aligned}
& \mathbf{E}_{L}^{N}\left(f, \operatorname{Av}_{j} h_{j}\right)^{2} \leq 2 \mathbf{E}_{L}^{N}\left[f, \operatorname{Av}_{\alpha} \mathbf{1}\left(\left|\bar{\eta}_{\alpha}\right| \leq \bar{n} k\right) \operatorname{Av}_{j \in \alpha} h_{j}\right]^{2}+ \\
+ & 2 \operatorname{Var}_{L}^{N}(f) \operatorname{Var}_{L}^{N}\left(\operatorname{Av}_{\alpha} F_{\alpha}\right),
\end{aligned}
$$

where $F_{\alpha}(\eta) \equiv F\left(\eta_{\alpha}\right)$ and

$$
F\left(x_{1}, \ldots, x_{k}\right) \equiv \mathbf{1}\left(\left|x_{1}+\cdots+x_{k}\right|>\bar{n} k\right) \frac{1}{k} \sum_{j=1}^{k} h\left(x_{j}\right) .
$$

Corollary 7.4 shows that:

$$
\operatorname{Var}_{L}^{N}\left(\operatorname{Av}_{\alpha} F_{\alpha}\right) \leq \frac{4 k}{L} \tilde{G}_{2}\left(F_{\alpha}, \rho\right)+o\left(L^{-1}\right) .
$$

But $\tilde{G}_{2}\left(F_{\alpha}, \rho\right)=o\left(k^{-1}\right)$ because:

$$
\left|\tilde{G}_{2}\left(F_{\alpha}, \rho\right)\right| \leq \operatorname{Var}^{\lambda_{\rho}}\left(F_{\alpha}\right) \leq 2\|h\|_{+\infty}^{2} \nu^{\lambda_{\rho}}\left(\left|\bar{\eta}_{\alpha}\right|>\bar{n} k\right)
$$

and Cramér's theorem (see for example Varadhan (1984)) can be used to estimate the last term. So there exists $\bar{k}(\beta, \varepsilon, \bar{\rho}, F)>0$ such that $\frac{4 k}{L} \tilde{G}_{2}\left(F_{\alpha}, \rho\right) \leq$ $\frac{\varepsilon}{2 L}$ for every $k>\bar{k}$. For every fixed $k>\bar{k}$ it is possible to find $L(\beta, \bar{\rho}, \varepsilon, k)$ so that for every $L>\bar{L}$ the last term in (7.18) is bounded from above by $\frac{\varepsilon}{2 L}$. In conclusion, (7.18) becomes $\operatorname{Var}_{L}^{N}\left(\operatorname{Av}_{\alpha} F_{\alpha}\right) \leq \frac{\varepsilon}{L}$. Using (7.17) we obtain (7.16).

STEP 2. Let $\bar{n}$ and $k$ be fixed positive integer. Then there exists $C(\beta, \bar{n}, k)>$ 0 such that:

$$
\begin{aligned}
& \mathbf{E}_{L}^{N}\left[f, \mathrm{Av}_{\alpha} \mathbf{1}\left(\left|\bar{\eta}_{\alpha}\right| \leq \bar{n} k\right) \mathrm{Av}_{j \in \alpha} h_{j}\right]^{2} \leq \\
\leq & C \mathcal{E}_{L}^{N}(f, f)+2 \mathbf{E}_{L}^{N}\left(f, \mathrm{Av}_{\alpha} \tilde{h}_{\alpha}\right)^{2},
\end{aligned}
$$

where:

$$
\tilde{h}_{\alpha} \equiv \mathbf{1}\left(\left|\bar{\eta}_{\alpha}\right| \leq \bar{n} k\right) \mathbf{E}_{L}^{N}\left(\operatorname{Av}_{j \in \alpha} h_{j} \mid \bar{\eta}_{\alpha}\right)
$$

Proof of Step 2. Jensen inequality yields:

$$
\begin{aligned}
& \mathbf{E}_{L}^{N}\left[f, \operatorname{Av}_{\alpha} \mathbf{1}\left(\left|\bar{\eta}_{\alpha}\right| \leq \bar{n} k\right) \operatorname{Av}_{j \in \alpha} h_{j}\right]^{2} \leq \\
\leq & 2 \mathbf{E}_{L}^{N}\left[f, \operatorname{Av}_{\alpha} \mathbf{1}\left(\left|\bar{\eta}_{\alpha}\right| \leq \bar{n} k\right) \operatorname{Av}_{j \in \alpha}\left(h_{j}-\mathbf{E}_{L}^{N}\left(h_{j} \mid \bar{\eta}_{\alpha}\right)\right)\right]^{2}+ \\
+ & 2 \mathbf{E}_{L}^{N}\left[f, \operatorname{Av}_{\alpha} \mathbf{1}\left(\left|\bar{\eta}_{\alpha}\right| \leq \bar{n} k\right) \operatorname{Av}_{j \in \alpha} \mathbf{E}_{L}^{N}\left(h_{j} \mid \bar{\eta}_{\alpha}\right)\right]^{2}
\end{aligned}
$$


We have to estimate the first term on the left hand side of (7.20). Denote by $\rho_{\alpha}$ the density $\bar{\eta}_{\alpha} / k$ in the interval $\alpha$ and define

$$
g_{\alpha} \equiv \operatorname{Av}_{j \in \alpha}\left(h_{j}-\mathbf{E}_{L}^{N}\left(h_{j} \mid \bar{\eta}_{\alpha}\right)\right)
$$

so that:

$$
\begin{aligned}
& \mathbf{E}_{L}^{N}\left[f, \operatorname{Av}_{\alpha} \mathbf{1}\left(\left|\bar{\eta}_{\alpha}\right| \leq \bar{n} k\right) \operatorname{Av}_{j \in \alpha}\left(h_{j}-\mathbf{E}_{L}^{N}\left(h_{j} \mid \bar{\eta}_{\alpha}\right)\right)\right]= \\
= & \operatorname{Av}_{\alpha} \mathbf{E}_{L}^{N}\left[f, \mathbf{1}\left(\left|\rho_{\alpha}\right| \leq \bar{n}\right) g_{\alpha}\right] .
\end{aligned}
$$

A simple computation shows that:

$$
\mathbf{E}_{L}^{N}\left[f, \mathbf{1}\left(\left|\rho_{\alpha}\right| \leq \bar{n}\right) g_{\alpha}\right]=\mathbf{E}_{L}^{N}\left[\mathbf{1}\left(\left|\rho_{\alpha}\right| \leq \bar{n}\right) \mathbf{E}_{L}^{N}\left(f, g_{\alpha} \mid \eta_{\alpha^{c}}\right)\right]
$$

Using Jensen and Schwarz inequalities we obtain:

$$
\begin{aligned}
& \mathbf{E}_{L}^{N}\left[f, \operatorname{Av}_{\alpha} \mathbf{1}\left(\left|\rho_{\alpha}\right| \leq \bar{n}\right) g_{\alpha}\right]^{2} \leq \\
\leq & 2|| h \|_{+\infty}^{2} \operatorname{Av}_{\alpha} \mathbf{E}_{L}^{N}\left[\mathbf{1}\left(\left|\rho_{\alpha}\right| \leq \bar{n}\right) \operatorname{Var}_{L}^{N}\left(f \mid \eta_{\alpha^{c}}\right)\right] .
\end{aligned}
$$

Now recall that $\operatorname{Var}_{L}^{N}\left(f \mid \eta_{\alpha^{c}}\right)=\operatorname{Var}_{\alpha}^{\bar{\eta}_{\alpha}}\left[f\left(\cdot \mid \eta_{\alpha^{c}}\right)\right]$ where $\mathbf{E}_{\alpha}^{\bar{\eta}_{\alpha}}(\cdot)$ is the expected value with respect to $\nu\left(\eta_{\alpha} \mid \bar{\eta}_{\alpha}\right)$. By Proposition 3.6 we know that there exists a positive constant $C_{1}(\beta, \bar{n}, k)$ such that:

$$
\begin{aligned}
& 1\left(\left|\rho_{\alpha}\right| \leq \bar{n}\right) \operatorname{Var}_{L}^{N}\left(f \mid \eta_{\alpha^{c}}\right)=\mathbf{1}\left(\left|\rho_{\alpha}\right| \leq \bar{n}\right) \operatorname{Var}_{\alpha}^{\bar{\eta}_{\alpha}}\left[f\left(\cdot \mid \eta_{\alpha^{c}}\right)\right] \leq \\
\leq & 1\left(\left|\rho_{\alpha}\right| \leq \bar{n}\right) C_{1} \mathcal{E}_{\alpha}^{\bar{\eta}_{\alpha}}\left(f\left(\cdot \mid \eta_{\alpha^{c}}\right), f\left(\cdot \mid \eta_{\alpha^{c}}\right)\right)= \\
= & 1\left(\left|\rho_{\alpha}\right| \leq \bar{n}\right) C_{1} \mathcal{E}_{\alpha}^{N-\bar{\eta}_{\alpha^{c}}}\left(f\left(\cdot \mid \eta_{\alpha^{c}}\right), f\left(\cdot \mid \eta_{\alpha^{c}}\right)\right)
\end{aligned}
$$

This inequality and (7.21) give:

$$
\mathbf{E}_{L}^{N}\left[f, \operatorname{Av}_{\alpha} 1\left(\left|\rho_{\alpha}\right| \leq \bar{n}\right) g_{\alpha}\right]^{2} \leq C_{2}(\beta, \bar{n}, k, F) \mathcal{E}_{L}^{N}(f, f)
$$

Using this estimate and (7.20) we have (7.19).

STEP 3. For every $\varepsilon>0$ there exists $\bar{n}(\beta, \bar{\rho})$ and $\bar{k}(\beta, \varepsilon, \bar{\rho}, F)>0$ such that for any $k>\bar{k}$ it is possible to find $\bar{L}(\beta, \bar{\rho}, \varepsilon, k)>0$ so that:

$$
\mathbf{E}_{L}^{N}\left(f, \mathrm{Av}_{\alpha} \tilde{h}_{\alpha}\right)^{2} \leq \frac{\varepsilon}{L} \operatorname{Var}_{L}^{N}(f),
$$

for every $L>\bar{L}$.

Proof of Step 3. Let $a, b \in \mathbb{R}$ be two constant to be fixed later. Define $\tilde{F}_{\alpha} \equiv \tilde{h}_{\alpha}-a-b\left(\rho_{\alpha}-\rho\right)$ where $\rho \equiv N / L$ and $\rho_{\alpha} \equiv \bar{\eta}_{\alpha} / k$. Obviously we have that:

$$
\mathbf{E}_{L}^{N}\left(f, \operatorname{Av}_{\alpha} \tilde{h}_{\alpha}\right)^{2} \leq \operatorname{Var}_{L}^{N}(f) \operatorname{Var}_{L}^{N}\left(\operatorname{Av}_{\alpha} \tilde{h}_{\alpha}\right)=\operatorname{Var}_{L}^{N}(f) \operatorname{Var}_{L}^{N}\left(\operatorname{Av}_{\alpha} \tilde{F}_{\alpha}\right)
$$

and Corollary 7.4 may be used to estimate the last variance. A simple calculation yields:

$$
\operatorname{Var}_{L}^{N}\left(\operatorname{Av}_{\alpha} \tilde{h}_{\alpha}\right) \leq 4 \frac{k}{L} \operatorname{Var}^{\lambda_{\rho}}\left(\tilde{F}_{\alpha}\right)+o\left(L^{-1}\right)
$$


We claim that we can choose $a$ and $b$ so that $\operatorname{Var}^{\lambda_{\rho}}\left(\tilde{F}_{\alpha}\right)=o\left(k^{-1}\right)$. If this is true by (7.23) we have immediately $(7.22)$ because for any $\varepsilon>0$ there exists $\bar{k}(\beta, \varepsilon, \bar{\rho}, F)$ such that for every $k>\bar{k}$ we have $k \operatorname{Var}^{\lambda_{\rho}}\left(\tilde{F}_{\alpha}\right)<\frac{\varepsilon}{2}$. For any such $k$ there exists $\bar{L}(\beta, \bar{\rho}, k)$ so that for every $L>\bar{L}(\beta, \bar{\rho}, k)$ the $o\left(L^{-1}\right)$ in (7.23) is bounded above by $\varepsilon / 2 L$.

It remains to show that $\operatorname{Var}^{\lambda_{\rho}}\left(\tilde{F}_{\alpha}\right)=o\left(k^{-1}\right)$. We notice that

$$
\tilde{h}_{\alpha}=\mathbf{1}\left(\left|\bar{\eta}_{\alpha}\right| \leq \bar{n} k\right) \mathbf{E}_{L}^{N}\left(\operatorname{Av}_{j \in \alpha} h_{j} \mid \bar{\eta}_{\alpha}\right)=\mathbf{1}\left(\left|\bar{\eta}_{\alpha}\right| \leq \bar{n} k\right) \mathbf{E}_{\alpha}^{\bar{\eta}_{\alpha}}\left(h_{1}\right)
$$

and that by Corollary 7.3 for $\left|\bar{\eta}_{\alpha}\right| \leq \bar{n} k$, the latter expectation may be rewritten as:

$$
\mathbf{E}_{\alpha}^{\bar{\eta}_{\alpha}}\left(h_{1}\right)=\mathbf{E}^{\lambda_{\rho_{\alpha}}}\left(h_{1}\right)+\frac{1}{k} G_{1}\left(h_{1}, \rho_{\alpha}\right)+o\left(k^{-1}\right)=g_{k}\left(\rho_{\alpha}\right)+o\left(k^{-1}\right),
$$

where:

$g_{k}\left(\rho_{\alpha}\right) \equiv \mathbf{E}^{\lambda_{\rho_{\alpha}}}\left(h_{1}\right)+\frac{1}{2 k}\left[\frac{\mathbf{E}^{\lambda_{\rho_{\alpha}}}\left(\eta_{1}, \eta_{1}, \eta_{1}\right) \operatorname{Var}^{\lambda_{\rho_{\alpha}}}\left(\eta_{1}\right)}{\operatorname{Var}^{\lambda_{\rho_{\alpha}}}\left(\eta_{1}\right)^{2}}-\frac{\mathbf{E}^{\lambda_{\rho_{\alpha}}}\left(\eta_{1}, \eta_{1}, h_{1}\right)}{\operatorname{Var}^{\lambda_{\rho_{\alpha}}}\left(\eta_{1}\right)}\right]$.

Clearly $g_{k}\left(\rho_{\alpha}\right)$ is a smooth function in the variable $\rho_{\alpha}$ and:

$$
\tilde{F}_{\alpha}=1\left(\left|\rho_{\alpha}\right| \leq \bar{n}\right) g_{k}\left(\rho_{\alpha}\right)-a-b\left(\rho_{\alpha}-\rho\right)+o\left(k^{-1}\right) .
$$

Take $a \equiv g_{k}(\rho), b \equiv g_{k}^{\prime}(\rho)$ and fix $\varepsilon^{\prime}>0$. Then:

$$
\begin{aligned}
& \operatorname{Var}^{\lambda_{\rho}}\left(\tilde{F}_{\alpha}\right) \leq \mathbf{E}^{\lambda_{\rho}}\left(\tilde{F}_{\alpha}^{2}\right)= \\
= & \mathbf{E}^{\lambda_{\rho}}\left[\tilde{F}_{\alpha}^{2} \mathbf{1}\left(\left|\rho_{\alpha}-\rho\right| \leq \varepsilon^{\prime}\right)\right]+\mathbf{E}^{\lambda_{\rho}}\left[\tilde{F}_{\alpha}^{2} \mathbf{1}\left(\left|\rho_{\alpha}-\rho\right|>\varepsilon^{\prime}\right)\right]= \\
= & \mathbf{E}^{\lambda_{\rho}}\left[\tilde{F}_{\alpha}^{2} \mathbf{1}\left(\left|\rho_{\alpha}-\rho\right| \leq \varepsilon^{\prime}\right)\right]+o\left(k^{-1}\right)
\end{aligned}
$$

where in the last line we used Cramér's theorem (see Varadhan (1984)). For $\varepsilon^{\prime}$ small enough and $\left|\rho-\rho_{\alpha}\right|<\varepsilon^{\prime}$, we can expand $g_{k}\left(\rho_{\alpha}\right)$ in Taylor series: $g_{k}\left(\rho_{\alpha}\right)=g_{k}(\rho)+g_{k}^{\prime}(\rho)\left(\rho-\rho_{\alpha}\right)+R_{k}$ where $\left|R_{k}\right| \leq C\left(\beta, \varepsilon^{\prime}\right)\left(\rho-\rho_{\alpha}\right)^{2}$. For $\varepsilon^{\prime}$ small enough and $\bar{n}>2|\rho|$ we have:

$$
\begin{aligned}
& \mathbf{E}^{\lambda_{\rho}}\left[\tilde{F}_{\alpha}^{2} \mathbf{1}\left(\left|\rho_{\alpha}-\rho\right| \leq \varepsilon^{\prime}\right)\right]= \\
= & \mathbf{E}^{\lambda_{\rho}}\left[\left(g_{k}\left(\rho_{\alpha}\right) \mathbf{1}\left(\left|\rho_{\alpha}\right| \leq \bar{n}\right)-a-b\left(\rho-\rho_{\alpha}\right)+o\left(k^{-1}\right)\right)^{2} \mathbf{1}\left(\left|\rho_{\alpha}-\rho\right| \leq \varepsilon^{\prime}\right)\right]= \\
= & \mathbf{E}^{\lambda_{\rho}}\left[\left(g_{k}\left(\rho_{\alpha}\right)-a-b\left(\rho-\rho_{\alpha}\right)+o\left(k^{-1}\right)\right)^{2} \mathbf{1}\left(\left|\rho_{\alpha}-\rho\right| \leq \varepsilon^{\prime}\right)\right]= \\
= & \mathbf{E}^{\lambda_{\rho}}\left[\left(R_{k}+o\left(k^{-1}\right)\right)^{2} \mathbf{1}\left(\left|\rho_{\alpha}-\rho\right| \leq \varepsilon^{\prime}\right)\right] \leq \mathbf{E}^{\lambda_{\rho}}\left[\left(R_{k}+o\left(k^{-1}\right)\right)^{2}\right] \leq \\
\leq & \mathbf{E}^{\lambda_{\rho}}\left[\left(C\left(\rho-\rho_{\alpha}\right)^{2}+o\left(k^{-1}\right)\right)^{2}\right] \leq 2 C^{2} \mathbf{E}^{\lambda_{\rho}}\left[\left(\rho-\rho_{\alpha}\right)^{4}\right]+o\left(k^{-1}\right)= \\
= & o\left(k^{-1}\right) .
\end{aligned}
$$

This concludes the proof. 
Now we can conclude the proof of Proposition 3.4. Fix $\varepsilon>0$ and $f \in L^{2}\left(\nu_{L}^{N}\right)$. By $(7.16)$ there exists $\bar{n}(\beta, \bar{\rho})$ and $\bar{k}(\beta, \varepsilon, \bar{\rho}, F)>0$ such that for every $k>\bar{k}$ it is possible to find $\bar{L}(\beta, \bar{\rho}, k)>0$ so that:

$$
\mathbf{E}_{L}^{N}\left(f, \mathrm{Av}_{j} h_{j}\right)^{2} \leq \frac{\varepsilon}{2 L} \operatorname{Var}_{L}^{N}(f)+2 \mathbf{E}_{L}^{N}\left[f, \mathrm{Av}_{\alpha} \mathbf{1}\left(\left|\bar{\eta}_{\alpha}\right| \leq \bar{n} k\right) \mathrm{Av}_{j \in \alpha} h_{j}\right]^{2},
$$

for any $L>\bar{L}$. Because of (7.19) the last term in this estimate is bounded above by

$$
2 C(\beta, \bar{n}, k) \mathcal{E}_{L}^{N}(f, f)+4 \mathbf{E}_{L}^{N}\left(f, \operatorname{Av}_{\alpha} \tilde{h}_{\alpha}\right)^{2} .
$$

Finally using (7.22) we obtain:

$$
\mathbf{E}_{L}^{N}\left(f, \mathrm{Av}_{j} h_{j}\right)^{2} \leq 2 C(\beta, \bar{n}, k) \mathcal{E}_{L}^{N}(f, f)+\frac{\varepsilon}{L} \operatorname{Var}_{L}^{N}(f)
$$

for $k=\bar{k}+1$ and $L>\bar{L}$.

\section{8. "A Priori" Estimates}

In this section we will prove Proposition 3.5 and 3.6. These are lower bounds on the spectral gap of some processes. The estimates we will obtain are not sharp, but this is not important for what they are used.

Proof of Proposition 3.5. We shall prove this proposition by induction on $L$.

Suppose $L=2$, then $\eta_{1}=N-\eta_{2}$ almost surely. Define for every $f \in L^{2}\left(\nu_{2}^{\delta, N}\right)$ the local function $\hat{f}_{N}\left(\eta_{2}\right) \equiv f\left(N-\eta_{2}, \eta_{2}\right)$, then $\operatorname{Var}_{2}^{\delta, N}(f)=$ $\operatorname{Var}_{2}^{\delta, N}\left(\hat{f}_{N}\right)$, and the Poincaré inequality (3.5) follows in this case from Proposition 3.1. Suppose now that (3.5) is true for a fixed $L \geq 2$. For every $f \in L^{2}\left(\nu_{L+1}^{\delta, N}\right)$ conditional variance formula gives:

$$
\operatorname{Var}_{L+1}^{\delta, N}(f)=\mathbf{E}_{L+1}^{\delta, N}\left[\operatorname{Var}_{L+1}^{\delta, N}\left(f \mid \eta_{L+1}\right)\right]+\operatorname{Var}_{L+1}^{\delta, N}\left[\mathbf{E}_{L+1}^{\delta, N}\left(f \mid \eta_{L+1}\right)\right]
$$

The proposition is proved if we can show that any term in this expression is bounded above by the form $\mathcal{E}_{L+1}^{\delta, N}(f, f)$ multiplied by a factor independent of $N$.

Recall that $\operatorname{Var}_{L+1}^{\delta, N}\left(f \mid \eta_{L+1}\right)=\operatorname{Var}_{L}^{\delta, N-\eta_{L+1}}\left[f\left(\cdot \mid \eta_{L+1}\right)\right]$ then induction assumption yields:

$$
\operatorname{Var}_{L}^{\delta, N-\eta_{L+1}}\left[f\left(\cdot \mid \eta_{L+1}\right)\right] \leq K(\beta, \delta, L) \mathcal{E}_{L}^{\delta, N-\eta_{L+1}}\left[f\left(\cdot \mid \eta_{L+1}\right), f\left(\cdot \mid \eta_{L+1}\right)\right] .
$$

This inequality, shows that the first term on the right hand side of (8.1) is bounded above by $K(\beta, \delta, L) \mathcal{E}_{L+1}^{\delta, N}(f, f)$. It remains to estimate the last term in (8.1). We can apply Proposition 3.1 to the local function $\mathbf{E}_{L+1}^{\delta, N}\left(f \mid \eta_{L+1}\right)$ to obtain:

$$
\operatorname{Var}_{L+1}^{\delta, N}\left[\mathbf{E}_{L+1}^{\delta, N}\left(f \mid \eta_{L+1}\right)\right] \leq C_{1}(\beta, \delta) \mathbf{E}_{L+1}^{\delta, N}\left[\left(\partial_{L+1}^{+} \mathbf{E}_{L+1}^{\delta, N}\left(f \mid \eta_{L+1}\right)\right)^{2}\right]
$$


Now (4.11) and a some simple estimates give:

$$
\begin{aligned}
& \operatorname{Var}_{L+1}^{\delta, N}\left[\mathbf{E}_{L+1}^{\delta, N}\left(f \mid \eta_{L+1}\right)\right] \leq \\
\leq & C_{2}(\beta, \delta)\left\{\mathbf{E}_{L+1}^{\delta, N}\left[\left(\partial_{L+1, L} f\right)^{2}\right]+\mathbf{E}_{L+1}^{\delta, N}\left[\operatorname{Var}_{L+1}^{\delta, N}\left(f \mid \eta_{L+1}\right)\right]\right\} .
\end{aligned}
$$

Again induction assumption shows that the last term in this expression is bounded above by $K(\beta, \delta, L) \mathcal{E}_{L}^{\delta, N}(f, f)$ so that:

$$
\operatorname{Var}_{L+1}^{\delta, N}\left[\mathbf{E}_{L+1}^{\delta, N}\left(f \mid \eta_{L+1}\right)\right] \leq C_{3}(\beta, \delta, L) \mathcal{E}_{L+1}^{\delta, N}(f, f)
$$

This concludes the proof.

In order to prove Proposition 3.6 we need a technical estimate on the ratio $\frac{\mathcal{E}_{L}^{N}(f, f)}{\operatorname{Var}_{L}^{N}(f)}$ :

Lemma 8.1. Suppose $f \in L^{2}\left(\nu_{L}^{N}\right)$ and define $f_{N}(\eta) \equiv f\left(\eta_{1}, \ldots, \eta_{L-1}, \eta_{L}+\right.$ N). Then:

$$
e^{-4 \beta N} \leq \frac{\operatorname{Var}_{L}^{N}(f)}{\operatorname{Var}_{L}^{0}\left(f_{N}\right)} \leq e^{4 \beta N} \quad e^{-2 \beta N} \leq \frac{\mathcal{E}_{L}^{N}(f, f)}{\mathcal{E}_{L}^{0}\left(f_{N}, f_{N}\right)} \leq e^{2 \beta N}
$$

for any $L>0$ and $N \in \mathbb{Z}$.

Proof. We can suppose $N \geq 0$. Define $h_{N}(x) \equiv e^{-\beta(|x+N|-|x|)}$ and notice that

$$
e^{-\beta N} \leq h_{N}(\eta) \leq e^{\beta N}
$$

This formula enable us to compare objects connected with $\nu_{L}^{N}$ for different values of $N$. For example:

$$
Z_{L}^{N}=\sum_{\eta} 1(\bar{\eta}=N) e^{-\beta \sum_{i=1}^{L}\left|\eta_{i}\right|}=\sum_{\eta} 1(\bar{\eta}=0) h_{N}\left(\eta_{1}\right) e^{-\beta \sum_{i=1}^{L}\left|\eta_{i}\right|}
$$

and by (8.3) we know that $e^{-\beta N} \leq Z_{L}^{N} / Z_{L}^{0} \leq e^{\beta N}$. A similar calculation shows that:

$$
\operatorname{Var}_{L}^{N}(f)=\left(\frac{Z_{L}^{0}}{Z_{L}^{N}}\right)^{2} \frac{1}{2} \sum_{\eta, \xi}\left[f_{N}(\eta)-f_{N}(\xi)\right]^{2} h_{N}\left(\eta_{1}\right) h_{N}\left(\xi_{1}\right) \nu_{L}^{0}(\eta) \nu_{L}^{0}(\xi)
$$

from which follows the first one of the estimates (8.2). The second one can be proved in a similar way.

Proof of Proposition 3.6. Notice that the map $f \mapsto f_{N}$ is a bijection of $L^{2}\left(\nu_{L}^{N}\right)$ onto $L^{2}\left(\nu_{L}^{0}\right)$. Lemma 8.1 implies that:

$$
\inf _{f \in L^{2}\left(\nu_{L}^{N}\right)} \frac{\mathcal{E}_{L}^{N}(f, f)}{\operatorname{Var}_{L}^{N}(f)} \geq e^{-6 \beta N} \inf _{f \in L^{2}\left(\nu_{L}^{0}\right)} \frac{\mathcal{E}_{L}^{0}(f, f)}{\operatorname{Var}_{L}^{0}(f)} \geq e^{-6 \beta \bar{\rho} L} \inf _{f \in L^{2}\left(\nu_{L}^{0}\right)} \frac{\mathcal{E}_{L}^{0}(f, f)}{\operatorname{Var}_{L}^{0}(f)} .
$$


so that we need only to estimate this last ratio. Define $H(\eta) \equiv \sum_{i=1}^{L}\left|\eta_{i}\right|$ and the jump rates:

$$
c\left(\eta \rightarrow \eta^{i, j}\right)= \begin{cases}1 & \text { if } H\left(\eta^{i, j}\right)<H(\eta) \\ e^{-2 \beta} & \text { if } H\left(\eta^{i, j}\right)>H(\eta) \\ 0 & \text { otherwise }\end{cases}
$$

where $\eta^{i, j} \equiv \eta-\delta_{i}+\delta_{j}$. The Markov generator defined as:

$$
(G f)(\eta)=\sum_{i, j} c\left(\eta \rightarrow \eta^{i, j}\right)\left[f\left(\eta^{i, j}\right)-f(\eta)\right]
$$

is a bounded and self adjoint in $L^{2}\left(\nu_{L}^{0}\right)$. Notice that:

$$
f\left(\eta^{i, j}\right)-f(\eta)=\sum_{k=j}^{i-1}\left(\partial_{k+1, k} f\right)\left(\eta^{i, k+1}\right)
$$

for every $i, j$ with $i>j$. This equation and an elementary calculation shows that:

$$
\mathcal{G}(f, f) \leq C_{1}(\beta, \delta) L^{3} \mathcal{E}_{L}^{0}(f, f)
$$

for every $f \in L^{2}\left(\nu_{L}^{0}\right)$. Here $\mathcal{G}(f, f)$ stands for the Dirichlet form associated with $G$. This inequality together with (8.4) shows that to prove the proposition we have to show that $\lambda_{1}(G)>0$ for every $L>0$. This fact is not obvious in our case because the state space of the Markov chain associated with $G$ is not finite. The technique we used to prove this lower bound can be found in Lawler, Sokal (1988). We consider a rooted graph $(V, E, \mathbf{0})$ where the vertex set $V$ and the edges set $E$ are defined as:

$$
\begin{aligned}
& V \equiv\left\{\eta \in \Omega_{L}: \bar{\eta}=0\right\} \\
& E \equiv\left\{(\eta, \xi) \in V \times V: \exists i, j \in \mathbb{Z} \text { such that } \xi=\eta^{i, j}\right\} .
\end{aligned}
$$

The root is the vertex $\mathbf{0} \equiv(0, \ldots, 0) \in V$. For every $\eta \in V$ we can define a geodetical path between $\eta \neq \mathbf{0}$ and $\mathbf{0}$ in the following way:

- Let $i_{1}$ be the first index such that $\eta_{i_{1}}>0$ and $j_{1}$ the first index such that $\eta_{j_{1}}<0$. Define $e_{1} \equiv\left(\eta, \eta^{i_{1}, j_{1}}\right) \in E ; e_{1}$ is the first edge in our path. If $\eta^{i_{1}, j_{1}}=\mathbf{0}$ then the geodetic path is $\left\{e_{1}\right\}$ else we repeat the procedure starting from the vertex $\eta^{i_{1}, j_{1}}$.

It is easy to convince ourselves that this procedure leads up to the construction of a geodetical path $\left\{e_{1}, e_{2}, \ldots, e_{k(\eta)}\right\}$. Notice that if $\eta(h) \in V$ is the first vertex of the edge $e_{h}$ then $H(\eta(h+1))=H(\eta(h))-2$ for every $h=1, \ldots, k(\eta)-1$. This means that the geodetical distance of $\eta$ from the root is $\frac{1}{2} H(\eta)$. We are in the setting of the random walk on a rooted graph treated in Lawler, Sokal (1988). By Corollary 5.5 in that paper we have that:

$$
\lambda_{1}(G) \geq \frac{1}{2 M z^{2}(2 \beta)}
$$

where $M \leq 2 L^{2}$ and $z(2 \beta) \leq[Z(2 \beta, 0)]^{L}$. This concludes the proof. 


\section{APPENDIX}

Lemma 9.1. Let $(\Omega, \mathcal{F}, \mu)$ be a probability space. Suppose that $f \in L^{2}(\mu)$ and $g \in L^{\infty}(\mu)$. Then for every $A \in \mathcal{F}$ such that $\mu(A)>0$ we have:

$$
\mathrm{E}(f, \mathbf{1}(A) g)^{2} \leq 2 \mathrm{E}(f, g \mid A)^{2}+8\|g\|_{+\infty}^{2} \mu\left(A^{c}\right) \operatorname{Var}(f) .
$$

Proof. By the definition of covariance we have:

$$
\begin{gathered}
\mathrm{E}(f, g)=\iint d \mu(x) d \mu(y) \mathbf{1}(x \in A) \mathbf{1}(y \in A)[f(x)-f(y)][g(x)-g(y)]+ \\
+\iint d \mu(x) d \mu(y)[1-\mathbf{1}(x \in A) \mathbf{1}(y \in A)][f(x)-f(y)][g(x)-g(y)] .
\end{gathered}
$$

A simple calculation yields:

$$
\begin{aligned}
\mathrm{E}(f, g)^{2} & \leq 2 \mu(A)^{4} \mathrm{E}(f, g \mid A)^{2}+8\|g\|_{+\infty} \operatorname{Var}\left(1\left(A^{c}\right)\right) \operatorname{Var}(f) \\
& \leq 2 \mathrm{E}(f, g \mid A)^{2}+8\|g\|_{+\infty} \mu\left(A^{c}\right) \operatorname{Var}(f) .
\end{aligned}
$$

By replacing $g$ with $\mathbf{1}(A) g$ in this formula we have $(9.1)$.

Lemma 9.2. Suppose $g=g\left(\eta_{1}\right) \in L^{\infty}\left(\nu_{L}^{N}\right)$, with $|N / L| \leq \bar{\rho}<+\infty$. Then:

$$
\mathbf{E}_{L}^{N+1}(g)-\mathbf{E}_{L}^{N}(g)=O\left(L^{-1}\right) .
$$

Proof. Define $\rho=\frac{N}{L}$ and $\rho^{\prime}=\frac{N+1}{L}$, the by Corollary 7.3 we have that:

$$
\mathbf{E}_{L}^{N+1}(g)-\mathbf{E}_{L}^{N}(g)=\mathbf{E}^{\lambda_{\rho^{\prime}}}(g)-\mathbf{E}^{\lambda_{\rho}}(g)+\frac{1}{L}\left[G_{1}\left(g, \rho^{\prime}\right)-G_{1}(g, \rho)\right]+o\left(L^{-1}\right) .
$$

It is easy to check that the term in square bracket is bounded, so we need only to show that $\mathbf{E}^{\lambda_{\rho^{\prime}}}(g)-\mathbf{E}^{\lambda_{\rho}}(g)=O\left(L^{-1}\right)$. This fact is true because an explicit computation shows that $\mathbf{E}^{\lambda_{\rho}}(g)$ is a smooth function of $\rho$, and $\rho^{\prime}=\rho+\frac{1}{L}$.

Lemma 9.3. There exists a positive constant $K(\beta)$ such that:

$$
\operatorname{Var}_{L}^{N}\left(\eta_{1}\right) \geq K\left(\frac{N^{2}}{L^{2}} \vee 1\right)
$$

Proof. We consider separately the two cases $|N / L| \leq$ cost. and $|N / L| \gg 1$. Fix $\bar{\rho}>0$, Corollary 7.3 gives:

$$
\operatorname{Var}_{L}^{N}\left(\eta_{1}\right)=\operatorname{Var}^{\lambda_{\rho}}\left(\eta_{1}\right)+O\left(L^{-1}\right)
$$

for $|N / L| \leq \bar{\rho}$. Here $\lambda_{\rho}$ is such that $\mathbf{E}^{\lambda_{\rho}}\left(\eta_{1}\right)=\rho \equiv N / L$ (see Lemma 6.1). We claim that $\lambda=0$ minimizes $\sigma^{2}(\lambda) \equiv \operatorname{Var}^{\lambda}\left(\eta_{1}\right)$. If this is true, by (9.3), there exists a constant $C_{1}(\beta)>0$ so that $\operatorname{Var}_{L}^{N}\left(\eta_{1}\right) \geq C_{1}$ for $|N / L| \leq \bar{\rho}$. The fact that $\lambda=0$ minimizes $\sigma^{2}(\lambda)$ is a trivial consequence of the fact that $\sigma^{2}(\lambda)$ is a positive convex symmetric function, as easily checked by an explicit calculation. 
The estimate $\operatorname{Var}_{L}^{N}\left(\eta_{1}\right) \geq C_{1}(\beta)>0$ is not a good estimate if $|N / L|$ is large. We claim that in general there exists a positive constant $C_{2}(\beta)$ such that:

$$
\operatorname{Var}_{L}^{N}\left(\eta_{1}\right) \geq C_{2} \frac{N^{2}}{L^{2}}
$$

The proof of (9.4) is by induction on $L$.

An explicit calculation shows that (9.4) holds for $L=2$. Assume that for a fixed $L>0$ that (9.4) holds for every $N \in \mathbb{Z}$. We shall show that:

$$
\operatorname{Var}_{L+1}^{N}\left(\eta_{1}\right) \geq C_{2} \frac{N^{2}}{(L+1)^{2}}
$$

for every $N \in \mathbb{Z}$. A simple calculation shows that

$$
\operatorname{Var}_{L+1}^{N}\left(\eta_{1}\right) \geq \mathbf{E}_{L+1}^{N}\left[\operatorname{Var}_{L}^{N-\eta_{L}}\left(\eta_{1}\right)\right]
$$

an by inductive hypothesis:

$$
\operatorname{Var}_{L+1}^{N}\left(\eta_{1}\right) \geq \frac{C_{2}}{L^{2}} \mathbf{E}_{L+1}^{N}\left[\left(N-\eta_{L}\right)^{2}\right]=\frac{C_{2}}{L^{2}} \mathbf{E}_{L+1}^{N}\left[\left(\eta_{1}-N\right)^{2}\right]
$$

Last term in this estimate may be explicitly calculated:

$$
\mathbf{E}_{L+1}^{N}\left[\left(\eta_{1}-N\right)^{2}\right]=\left(\frac{N L}{L+1}\right)^{2}+\operatorname{Var}_{L+1}^{N}\left(\eta_{1}\right),
$$

and finally by (9.6):

$$
\operatorname{Var}_{L+1}^{N}\left(\eta_{1}\right)\left(1-\frac{C_{2}}{L^{2}}\right) \geq \frac{C_{2} N^{2}}{(L+1)^{2}}
$$

This relation implies $(9.5)$.

I would like to thank F. Martinelli who posed me this problem and helped me with many constructive discussions. I would also like to thank H.T. Yau for the enlightening discussion we had in Rome in the Spring of 1994.

\section{ReFERENCES}

Dobrushin, R., Kontecký, R. and Shlosman, S. (1992), Wulff Construction. A Global Shape from Local Interaction, Translation of Mathematical Monographs, Vol. 104, AMS.

Landim, C., Sethuraman, S. and Varadhan, S. R. S. (1995), Spectral Gap for ZeroRange Dynamics, Preprint to appear Ann. Probab.

Lawler, G. F. and Sokal, A. D. (1988), Bounds on the $L^{2}$ Spectrum for Markov Chains and Markov Processes: a Generalization of Cheeger's Inequality, Trans. Amer. Math. Soc, 309 No $2557-580$.

Lu, S. and YAU, H.T. (1993), Spectral Gap and Logarithmic Sobolev Inequality for Kawasaki and Glauber Dynamics, Commun. Math. Phys. 156 399-433.

Petrov, V. V. (1975), Sums of Independent Random Variables. Springer-Verlag.

Varadhan, S. R. S. (1984), Large Deviation and Application, Society for Industrial and Applied Mathematics.

YAU, H. T. (1994), Logarithmic Sobolev Inequality for Kawasaki Dynamics, I. the Independent Case, Preprint.

Istituto G. Castelnuovo, Università "La Sapienza" P.le A. Moro 2, 00185 Roma, Italy. EmaIL: postagusemat.uniroma1.it. 